\title{
A Review of Recent Results on Ocean Acoustic Wave Propagation in Random Media: Basin Scales
}

\author{
John A. Colosi and the ATOC Group
}

(Invited Paper)

\begin{abstract}
Measurements of basin-scale acoustic transmissions made during the last four years by the Acoustic Thermometry of Ocean Climate (ATOC) program have allowed for the study of acoustic fluctuations of low-frequency pulse propagation at ranges of 1000 to $5000 \mathrm{~km}$. Analysis of data from the ATOC Acoustic Engineering Test conducted in November 1994 has revealed new and unexpected results for the physics of ocean acoustic wave propagation in random media. In particular, use of traditional $\Lambda, \Phi$ methods (using the Garrett-Munk (GM) internal wave model) to identify the wave propagation regime for early identifiable wavefronts predict the saturated regime, whereas observations of intensity probability density functions, intensity variance, and pulse time spread and wander suggest that the propagation is more likely near the border between the unsaturated and partially saturated regimes. Calculations of the diffraction parameter $\Lambda$ are very sensitive to the broad-band nature of the transmitted pulse, with $\mathrm{CW}$ calculations differing from a simplistic broad-band calculation by $10^{3}$ ! A simple model of pulse propagation using the Born approximation shows that CW and broad-band cases are sensitive to a random medium very differently and a theoretical description of broad-band effects for pulse propagation through a random media remains a fundamental unsolved problem in ocean acoustics.

The observations show that, at $75-\mathrm{Hz}$ center frequency, acoustic normal mode propagation is strongly nonadiabatic due to random media effects caused by internal waves. Simulations at a lower frequency of $28 \mathrm{~Hz}$ suggest that the first few modes might be treated adiabatically even in a random ocean. This raises the possibility of using modal techniques for ocean acoustic tomography, thereby increasing the vertical resolution of thermometry.

Finally, the observation of unsaturated or partially saturated propagation for $75-\mathrm{Hz}$ broad-band transmissions, like those of ATOC, suggests that ray-based tomography will be robust at basin-scales. This opens up the possibility of ray-based internal wave tomography using the observables of travel time variance, and vertical and temporal coherence. Using geometrical optics and the GM internal wave spectrum, internal wave tomography
\end{abstract}

Manuscript received August 6, 1998; revised December 31, 1998. This work was supported by the Office of Naval Research (ONR) ocean acoustics program and supported in part by the Strategic Environmental Research and Development Program through Defense Advanced Research Projects Agency (DARPA) under Grant MDA 972-93-1-0003. The work of J. A. Colosi was supported by Office of Naval Research Young Investigator Award, from the J. Lamar Worzel Assistant Scientist Fund, and by the Penzance Endowed Fund in Support of Scientific Staff. This is Woods Hole Oceanographic Institution contribution number 9769.

J. A. Colosi is with Woods Hole Oceanographic Institution, Woods Hole, MA 02543 USA.

The members of the Acoustic Thermometry of Ocean Climate (ATOC) Group are A. B. Baggeroer, T. G. Birdsall, C. Clark, B. D. Cornuelle, D. Costa, B. D. Dushaw, M. A. Dzieciuch, A. M. G. Forbes, B. M. Howe, D. Menemenlis, J. A. Mercer, K. Metzger, W. Munk, R. C. Spindel, P. F. Worcester, and C. Wunsch.

Publisher Item Identifier S 0364-9059(99)03039-3. for an assortment of parameters of the GM model can be formulated in terms of a mixed linear/nonlinear inverse. This is a significant improvement upon a Monte Carlo approach presented in this paper which is used to infer average internal wave energies as a function of depth for the SLICE89 experiment. However, this Monte Carlo approach demonstrated, for the SLICE89 experiment, that the GM model failed to render a consistent inverse for acoustic energy which sampled the upper $100 \mathrm{~m}$ of the ocean. Until a new theory for the forward problem is advanced, internal wave tomography utilizing the signal from strong mode coupling can only be carried out using time-consuming Monte Carlo methods.

Index Terms-Acoustic propagation, internal waves, scattering, tomography.

\section{INTRODUCTION}

$\mathbf{T}$ HE preponderance of effort to understand ocean acoustic wave propagation in random media (WPRM) has been focused on relatively high acoustic frequency and short-range experiments [1]-[3]. In the 1990s, low-frequency basin-scale experiments were motivated by the desire to measure ocean climate change [4], and acoustical oceanographers undertook the design of their experiments by extrapolating the established WPRM ideas to low frequency and long range. At the same time, the possibility of using acoustic fluctuations to tomographically infer internal wave energies and spectra was intriguing. It was far from obvious, however, that the signals would not be seriously degraded after propagating across an ocean basin. The 1000-km SLICE89 transmission experiment, which revealed strong internal-wave-induced acoustic scattering in the pulse crescendo, generated the following questions for basin-scale transmissions: would the acoustic scattering from internal waves be too strong to identify stable arrivals? Would the acoustic propagation be adequately described by geometrical optics so that ocean acoustic tomography could be effective? Could phase coherent processing be used on longrange signals to detect the signals and achieve the necessary signal-to-noise ratios? As it has turned out, much has been learned from recent basin-scale experiments, and this is the subject of this paper.

Instrumentation of the northeastern Pacific was carried out by the Acoustic Thermometry of Ocean Climate (ATOC) program. Since December of 1995, ATOC has been transmitting pulses at a center frequency of $75 \mathrm{~Hz}$ from a bottommounted source $($ depth $=930 \mathrm{~m})$ on Pioneer Seamount to several receivers throughout the north Pacific (Fig. 1). 


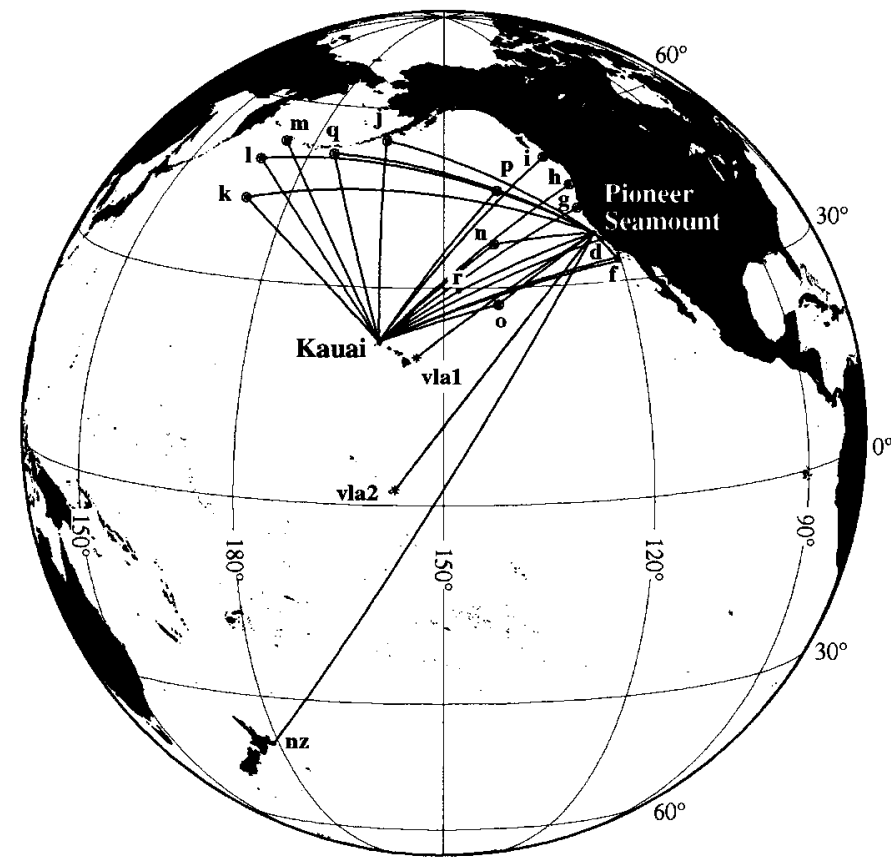

Fig. 1. ATOC transmission paths from the Pioneer Seamount and the Kauai sources. Alphabetic symbols are for bottom-mounted horizontal arrays. VLA's are labeled VLA1 and VLA2.

Receivers include two moored 40-element vertical line arrays (VLA's) spanning $1400 \mathrm{~m}$ (VLA1 and VLA2) and several bottom-mounted horizontal arrays (alphabetic labels; see [5] for a discussion of data from these receivers). Signals are sent every $4 \mathrm{~h}$ during transmission periods established by the ATOC Marine Mammal Research Program.

An example of a wavefront measured at the Hawaii VLA is shown in Fig. 2 together with two numerical simulations. The single pulse shown in Fig. 2 consists of 40 repeated $m$ sequences, which are coherently averaged to increase signalto-noise levels [6]. The energy which arrives at the earlier travel times shows fronts that form a double accordion pattern. This is the ray-like region. The ray-like region contains the data for a typical ocean acoustic tomography analysis because every point along the front can be associated with a geometrical optics ray that samples the ocean in a specific way. The earliest arrivals are composed of rays that reflect from the ocean surface and sample nearly the whole water column. Later fronts are composed of rays that have upper turning points in the upper $300 \mathrm{~m}$ of the ocean. The region at late arrival times in which ray-like fronts are no longer apparent is called the mode-like region. The mode-like region is composed of acoustic energy that is trapped near the sound-channel axis and can be described by low-order acoustic modes. Note that the typical double accordion pattern of the arrival as seen in the simulations is not evident in the ATOC data. This is a result of bottom acoustic energy stripping near the source which eliminates initially downward propagating energy.

The upper panel of Fig. 2 shows a time front from a numerical simulation with internal wave sound-speed perturbations obeying the Garrett-Munk (GM) internal wave spectrum [7], [8]; the center panel is a measured pulse from the ATOC
Hawaii VLA, and the lower panel is a numerical simulation without internal wave sound-speed perturbations. For the early ray-like region, the three time fronts are qualitatively the same: the data and the simulation with internal waves have small time shifts due to internal waves and the intensity varies along the fronts. In the mode-like region, however, the data and the simulation with internal waves show significant broadening of the energy in depth compared with the case of no internal waves. It is in this region that the internal waves show their largest effect; this can be understood in terms of mode coupling [9] or ray refraction [10], [11]. The general arrival pattern as just described is now known to be typical of low-frequency, long-range, and broad-band acoustic transmissions in the ocean [11], [6].

The primary focus of this paper will be on an experiment conducted by the ATOC program in November of 1994 called the Acoustic Engineering Test (AET) in which signals with a $75-\mathrm{Hz}$ center frequency and a bandwidth of $37.5 \mathrm{~Hz}(3-\mathrm{dB}$ point) were transmitted from a source suspended from $\mathrm{R} / \mathrm{P}$ FLIP, which was moored roughly $250 \mathrm{~nm}$ south-southwest of San Diego near Jasper Seamount [6]. The signals were received on two 20-element 700-m long VLA's; one located approximately $85 \mathrm{~km}$ from the source and one located $3250 \mathrm{~km}$ away near the island of Hawaii. Signals were also observed on several bottom-mounted horizontal arrays (see [5]). Transmissions were carried out over a 6-day period with a 2-h minimum time separation between transmissions. The AET showed acoustic fluctuations consistent with unsaturated or nearly partially saturated wave field statistics based on observations of the intensity probability density function (PDF), intensity variance, and the pulse time spread and wander [13]. Predictions of the wave propagation regime based on traditional $\Lambda$ and $\Phi$ calculations [1], [2] indicate strong saturated behavior in contradiction to the observations. In addition, predictions of pulse time spread $\tau_{0}$, using the theory of Dashen and Flatté [12], are larger than the observations by nearly two orders of magnitude [13]. In this paper, it is argued that the failure of these theories to explain the AET is due to CW or narrow-band assumptions in the theory.

This failure and the past successes of this body of theory, even for pulse propagation exeriments, are associated with a few facts. First, only recently has it been possible to exactly calculate the acoustic weighting function for propagation through internal waves along a geometrical optics ray path [13]. Previous approximations have been shown to be in significant error by placing too much weight at the ray upper apex and not enough weight several hundreds of meters below the apex [13]. This effect does not have a strong impact on the calculation of $\Phi$ but it has a strong influence on the calculation of $\Lambda$ and $\tau_{0}$. Also, comparisons have not been made with observations for a very long propagation range; previous theory/observation comparisons were for short range and higher acoustic frequency and in general the acoustic fluctuations were small. The very long range of the AET and the strong range scaling of the theory have made the differences apparent.

As support for this argument on broad-band effects, a calculation of the ray-tube extent for a constant sound-speed 


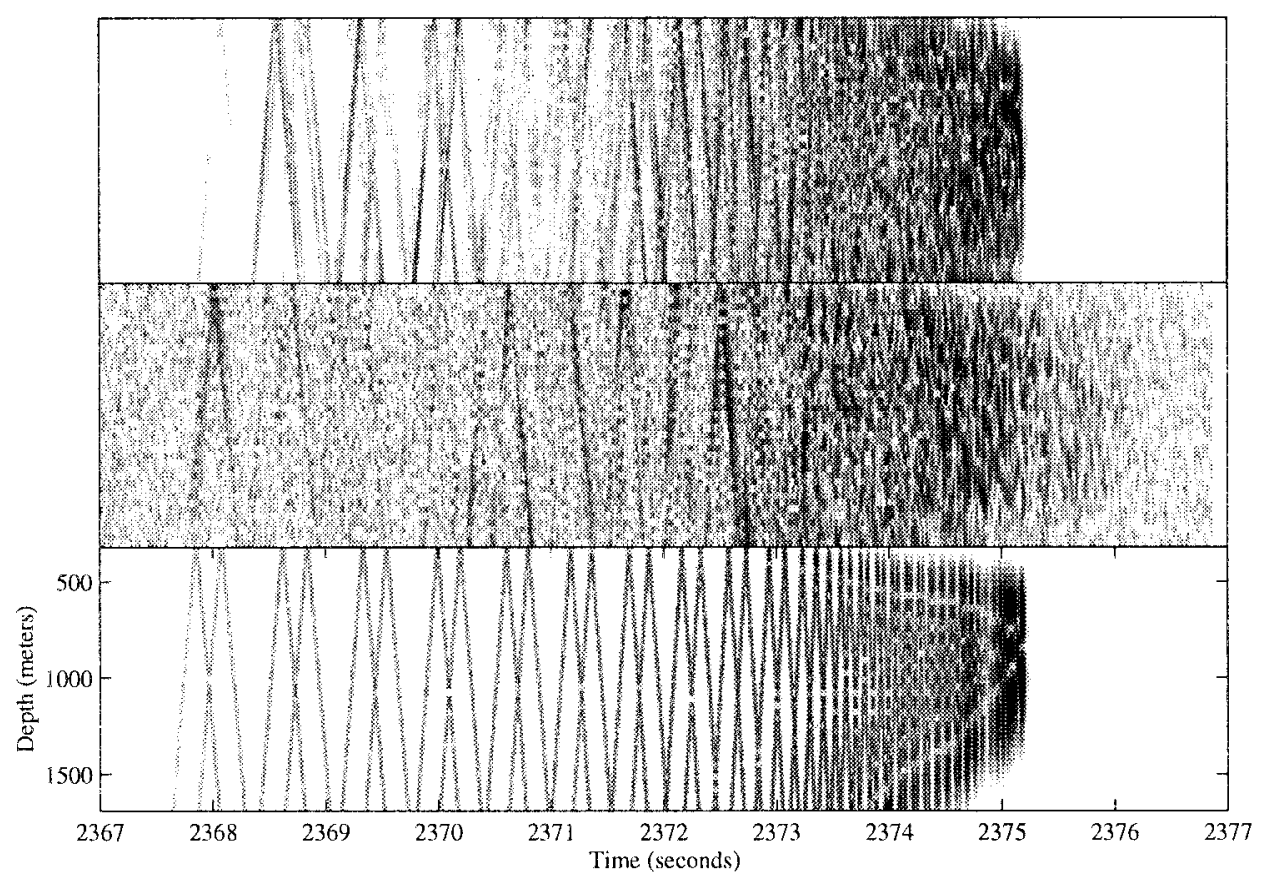

Fig. 2. Time fronts from the ATOC VLA1 and numerical simulations. The upper panel is a simulation using the Levitus 1994 sound-speed database plus internal wave sound-speed perturbations at the GM reference level. The middle panel is a measured time front, and the lower panel is a simulation without internal waves.

channel is presented. This approach is similar to that of Bowlin who showed how to calculate ray tubes for arbitrary sound-speed profiles [14]. For finite frequencies and broadband signals, it is shown that ray paths have finite spatial extent and that this spatial extent is the characteristic scale of the broad-band acoustic field. This is in contrast to $\mathrm{CW}$ signals whose characteristic spatial extent is the first Fresnel zone radius $\left(R_{f}\right)$. For the practical cases presented in this paper, the ray-tube extent $\xi$ is constant along a ray path and is closely given by $\xi=c_{0} / \alpha$ where $\alpha$ is the signal bandwidth. Replacing $R_{f}$ with $\xi$ in the definition of the diffraction parameter $\Lambda$ gives broad-band values of $\Lambda$ for the AET that are smaller than the CW $\Lambda$ by a factor of nearly 1000 . This approach is obviously ad hoc since the original derivation of $\Lambda$ for $\mathrm{CW}$ cases was based on the physics of the Rytov theory [1]. The approach here is heuristic to underscore the differences in the characteristic spatial scales of the acoustic field, with the understanding that the characteristic scales have important physical meaning. To examine the physics more closely, a treatment of pulse propagation using the wellknown Born approximation is presented where the limiting case of a CW signal can be seen. For a broad-band signal, the important parameter is $q=\alpha / \sigma_{0}$, the bandwidth divided by the center frequency. It is shown that for broad-band signals, like the ATOC signal $(q=0.6)$, pulse scattering is indeed very different than $\mathrm{CW}$ scattering. In particular, pulses are insensitive to scales larger than the first Fresnel zone radius at the center frequency where as $\mathrm{CW}$ signals have no such cutoff. The broad-band effects shown here, however, are not a factor of 100 or 1000 effect as would be required to describe the AET results. This suggests that more work is needed to understand the AET results, but this is a first step toward a fully broad-band theory of ocean acoustic WPRM.
Observations are also presented which show strong internalwave-induced scattering in the final arrivals that reduces the number of resolved wavefronts in basin-scale transmissions, thereby reducing the vertical resolution possible for tomography. Observed low-order acoustic mode arrivals from the ATOC transmissions to Kiritimati and Hawaii (see Fig. 1) are strongly nonadiabatic so that vertical resolution cannot be improved by adding mode travel times to the tomography inverse. Numerical simulations presented in this paper suggest that lower frequencies, on the order of $30 \mathrm{~Hz}$, may result in sufficient adiabaticiy for the lowest order modes to be used for tomography.

The observation of unsaturated or partially saturated propagation suggests that ray-based tomographic techniques for large scales (ocean acoustic tomography) and small scales (internal wave tomography) are robust. A scheme is outlined for performing ray-based inversions for internal wave model spectral parameters based upon observations of travel time variance, and time and depth coherence. Also presented are inversions for average internal wave displacement variance as a function of depth for the SLICE89 experiment [15], [9], based on Monte Carlo forward modeling using the GM internal wave spectrum, and observations of wavefront travel-time fluctuations and vertical scattering of acoustic energy in the wavefront finale. It is found for the SLICE89 experiment that the GM model cannot yield a consistent inverse for internal wave displacement variance for acoustic energy, which has sampled the upper $100 \mathrm{~m}$ of the ocean. This result reveals the well-known oceanographic fact that the GM model is not an upper ocean model; in fact, it is surprising that the GM model works to a depth of $100 \mathrm{~m}$ !

This paper is organized as follows. Section II discusses the theory of WPRM regimes as described by unsaturated, 
partially saturated, and fully saturated wave fields and presents observations and predictions for the AET. Section II also discusses broad-band effects for ocean acoustic WPRM. Random media effects related to large-scale tomography and internal wave tomography are examined in Section III. A summary and discussion are given in Section IV.

\section{BASIN-SCALE WPRM}

The AET experiment conducted in the eastern North Pacific by the ATOC program has revealed some unexpected results relating to the nature of acoustic wave propagation regimes in a fluctuating ocean in terms of unsaturated, partially saturated, and saturated wave fields. The nature of wave propagation in a random medium as a function of range, frequency, and spectra of wave speed fluctuations has been expressed using the parameters $\Phi$ and $\Lambda$, which will be discussed below [1], [2]. The $\Lambda, \Phi$ expressions which have been used in the past to predict unsaturated to saturated wave propagation regimes completely fail for the AET [13]. In addition, predictions of pulse time spread $\tau_{0}$, using the path integral theory of Dashen and Flatté [12], overpredict the spread by nearly two orders of magnitude [13]. Furthermore, it is asserted that the failure is due to the $\mathrm{CW}$ and narrow-band assumptions inherent in the treatment of $\Lambda$ and the pulse spread, and that a broad-band theory is needed to describe pulse transmission experiments like the AET.

A discussion of acoustic fluctuation quantities is given first to provide some basic definitions and provide a framework for the interpretation of the AET results.

\section{A. Acoustic Fluctuation Quantities}

The strength parameter $\Phi$ represents, in the geometrical optics limit, the rms phase fluctuation of a ray for a fixed ray path $\Gamma=z_{r}(x)$. This quantity is given by

$$
\Phi^{2}=\sigma^{2} \int_{\Gamma} d s \frac{\left\langle\mu^{2}\left(z_{r}\right)\right\rangle L_{p}\left(\theta, z_{r}\right)}{c^{2}}
$$

where $\left\langle\mu^{2}\left(z_{r}\right)\right\rangle$ is the fractional sound-speed variance, $\sigma$ is the acoustic frequency, and $L_{p}$ is the effective correlation length of the internal waves along the ray path $\Gamma . L_{p}$ is calculated using the expression

$$
\begin{array}{r}
L_{p}\left(s_{1}\right)=\frac{1}{\left\langle\mu^{2}\left(s_{1}\right)\right\rangle}\left[\left(\frac{d c}{d z}\right)_{p}\right]_{1} \int_{\Gamma_{0}} d s_{2}\left[\frac{1}{c^{2}}\left(\frac{d c}{d z}\right)_{p}\right]_{2} \\
\times \rho(\Delta x, \Delta z ; \bar{z})
\end{array}
$$

where $\rho(\Delta x, \Delta z ; \bar{z})=\left\langle\zeta\left(s_{1}\right) \zeta\left(s_{2}\right)\right\rangle$ is the correlation function of internal wave displacements. Note that the Markov approximation has not been made in our evaluation of $L_{p}$, which is extremely important to accurate predictions [13]. This approach has also been taken by Henyey [3] in examining the validity of the Markov approximation for steeply turning rays.

The diffraction parameter $\Lambda$ is the weighted average of $\left(R_{f}^{2}\left\{k_{v}^{2}\right\}\right) /(2 \pi)$ along a ray, where $R_{f}(x)$ is the first Fresnel zone radius and $\left\{k_{v}^{2}\right\}$ is the spectrum-weighted average value of the square of the vertical wavenumber of internal waves.
Thus,

$$
\Lambda=\Phi^{-2} \sigma^{2} \int_{\Gamma} d s \frac{\left\langle\mu^{2}\left(z_{r}\right)\right\rangle L_{p}\left(\theta, z_{r}\right)}{c^{2}} \frac{\left\{k_{v}^{2}\right\} R_{f}^{2}}{2 \pi}
$$

where $R_{f}^{2}(x)=\lambda|g(x, x)|, \lambda$ is the acoustic wavelength, and the Green's function [2] $g\left(x, x^{\prime}\right)$ gives the vertical distance from a ray to a nearby "broken" ray having unit slope discontinuity at $x=x^{\prime} . \Lambda$ is also closely linked to the variance of log-intensity $\left(\sigma_{\ln I}^{2}\right)$. For $\Lambda \ll 1$, Flatté et al. [1] have shown from Rytov theory and a GM-like fluctuation spectrum that $\sigma_{\ln I}^{2} \propto \Lambda \Phi^{2}$ where the proportionality constant is very close to one. For $\Lambda \gg 1$, Flatté et al. [1] have shown that $\sigma_{\ln I}^{2} \propto \Phi^{2} / 2$.

The average pulse shape is an important measure of acoustic fluctuations, since sound-speed variations can distort the pulse in the saturated and partially saturated regimes. The Fourier transform of the mutual coherence function of frequency combined with the appropriate source transfer function, $P(\Delta \sigma)$, gives the ensemble averaged pulse (EAP)

$$
\langle I(t)\rangle=\int_{-\infty}^{\infty} d \Delta \sigma P(\Delta \sigma)\left\langle\psi^{*}(\Delta \sigma) \psi(0)\right\rangle e^{i \Delta \sigma t} .
$$

Following Dashen and Flatté [12], [16], in the case of full saturation, the mutual coherence function for small frequency separations $\Delta \sigma$ can be written as

$$
\left\langle\psi^{*}(\Delta \sigma) \psi(0)\right\rangle \simeq \exp \left[-\frac{(\Delta \sigma)^{2}}{2}\left(\tau^{2}+\tau_{0}^{2}\right)\right] \cdot \exp \left(i \Delta \sigma \tau_{1}\right)
$$

with

$$
\begin{aligned}
\tau^{2}= & \Phi^{2} / \sigma^{2} \\
\tau_{1}= & \frac{\ln (\Phi)}{2 c_{0}} \int_{\Gamma} d s\left\langle\mu^{2}(z)\right\rangle L_{p}(\theta, z)\left\{k_{v}^{2}\right\} g(x, x) \\
\tau_{0}^{2}= & \left(\frac{\ln (\Phi)}{2 c_{0}}\right)^{2} \int_{\Gamma} d s\left\langle\mu^{2}(z)\right\rangle L_{p}(\theta, z)\left\{k_{v}^{2}\right\} \\
& \times \int_{\Gamma} d s^{\prime}\left\langle\mu^{2}\left(z^{\prime}\right)\right\rangle L_{p}\left(\theta^{\prime}, z^{\prime}\right)\left\{k_{v}^{2}\right\}\left[g\left(x, x^{\prime}\right)\right]^{2} \\
\tau_{0}^{2} \simeq & \tau_{1}^{2} .
\end{aligned}
$$

The parameters $\tau, \tau_{0}$, and $\tau_{1}$ can be interpreted as follows. The quantities $\tau^{2}$ and $\tau_{0}^{2}$ combine in quadrature to give the width of the pulse while $\tau_{1}$ represents a shift of the mean pulse arrival time (i.e., a travel-time bias). The quantity $\tau^{2}$ represents the travel-time variance that one would measure in the unsaturated region. The quantity $\tau_{0}^{2}$ represents the effect of pulse spreading due to loss of coherence between frequencies. Physically this coherence loss can be understood as a result of the interference of many uncorrelated micro-rays. Also of interest is that $\tau_{0}$ and $\tau_{1}$ both scale roughly like the square of the range [16].

\section{B. Wave Propagation Regimes}

A qualitative understanding of the different wave propagation regimes is needed to interpret the results presented here. The approach has been to divide the $\Lambda-\Phi$ space up into regions describing saturated, unsaturated, and partially saturated wave field statistics with the understanding that the border regions between regimes are not absolutely defined [1]. 
1) Unsaturated Region: For $\Lambda<1$ and $\Lambda \Phi^{2}<1$, wave propagation is adequately described by geometrical optics and is called the unsaturated region. In the unsaturated region, the acoustic field from an impulsive source as measured at a receiver can be understood in terms of a single geometrical optics ray path where the received pulse is just a replica of the transmitted pulse. In the unsaturated region, travel time and phase are precisely related; that is, the variance of travel time $\tau^{2}=\Phi^{2} / \sigma^{2}$. Intensity fluctuations result from weak focusing and defocusing of the ray tube; the $\Lambda \Phi^{2}<1$ boundary suggests that $\sigma_{\ln I}^{2}$ is less than one (i.e., rms 4.3$\mathrm{dB}$ fluctuations). However, the Rytov theory, from which this result is derived, is known only to be accurate for $\sigma_{\ln I}^{2}<0.3$ [17], [18]. Also, the log of intensity is normally distributed, as can be seen from the weak fluctuation theory of Rytov [1], [19].

2) Full Saturation: For $\Lambda \Phi>1$ and $\Phi>1$, wave propagation is called fully saturated. In the transition from geometrical optics to full saturation, the single geometrical optics path fractures into several uncorrelated micro-rays whose travel times are very close to the travel time of the original ray. Since the micro-rays have different travel times, the original pulse shape is not observed at the receiver; the pulse is spread in time. In the fully saturated regime, pulse time spread $\tau_{0}$ is larger than the travel-time variance $\tau$. Phase and travel time are not simply related and, if the acoustic field is considered to be a phasor, then this phasor executes a random walk in the phase plane. Intensity fluctuations resulting from the complex interference pattern of the micro-rays yield an exponential intensity PDF, and $\sigma_{\ln I}^{2}$ approaches $\pi^{2} / 6(\mathrm{rms}$ $5.56 \mathrm{~dB}$ ). However, the approach to the exponential PDF as $\Lambda \Phi$ increases is known to be quite slow [20].

3) Partial Saturation: For $\Lambda \Phi<1$ and $\Lambda \Phi^{2}>1$, wave propagation is called partially saturated. In this case, microrays are created by the small-scale fluctuations in the medium, but they are correlated with one another by the large-scale fluctuations. This region is difficult to analyze since one must know the details of the micro-ray coherence to calculate quantities such as travel-time variance, pulse spread, and intensity distribution. In the partially saturated regime, the pulse time spread and the travel-time variance may be comparable, and $\sigma_{\ln I}^{2}$ can reach its maximum values. This latter fact is why the partial saturation regime is often called the strong focusing region [20].

\section{AET Results}

With the previous background material, a discussion of the AET results can proceed. These results have been been documented in detail by Colosi et al. [13] and Worcester et al. [6]. For the AET transmissions, the observed travel-time variances $\tau^{2}$ are between $(11 \mathrm{~ms})^{2}$ and $(19 \mathrm{~ms})^{2}$, indicating rms phase variations in excess of $2 \pi$. The agreement between the predictions using (1) and the GM internal wave model at one-half the reference energy and the observations is very good [13].

The observed pulse spreads $\tau_{0}$ are much smaller than $\tau$ with values between 0 and $5 \mathrm{~ms}$ rms. Predictions of pulse spread using (7) and (9) are not in agreement with the observations [13]. The predictions give spread estimates that are two orders of magnitude too large. It should be noted also that the predictions from (7) do not match the variablity of the spread as a function of ray turning point depth. The narrow-band calculation predicts that shallower-turning rays should have more spread than deeper-turning rays [13].

In terms of intensity behavior for the AET, early arriving wavefronts have intensity fluctuations which are clearly not described by the exponential distribution of full saturation but are closer to a log-normal distribution more typical of the unsaturated regime [12]. Observed variances of log-intensity $\sigma_{\ln I}^{2}$ are between 0.21 and 0.51 , which are close to and in excess of the weak fluctuation limit of 0.3 .

Collectively, the AET observations show behavior typical of unsaturated or barely partially saturated propagation. This conclusion is supported by the facts that: 1) $\tau_{0} \ll \tau$; 2) the PDF of intensity is close to a log-normal distribution; and 3) $\sigma_{\ln I}^{2}$ is close to but slightly larger than weak fluctuation values.

On the other hand, predictions of the wave propagation regime using $\Lambda$ and $\Phi$ (see Fig. 4) predict that all of the rays for the AET are well within the saturated region, where the pulse is expected to be a complex interference pattern of uncorrelated micro-rays. In full saturation $\tau_{0} \gg \tau$, the intensity PDF is expected to be close to exponential, and $\sigma_{\ln I}^{2}$ should be close to $\pi^{2} / 6$ (i.e., 1.64). Clearly, none of these conditions are satisfied by the data. This is a completely unexpected result.

\section{Broad-Band versus Narrow-Band and CW Calculations}

The unexpected AET results suggest a fundamental problem with the theory of acoustic fluctuations for basin-scale transmissions like those of ATOC. Since it is certain that the GM internal wave model is at least a zeroth-order description of ocean internal waves, the wave propagation physics must be scrutinized. In particular, the parameters $\Lambda$ and $\tau_{0}$ as defined in (3) and (9) are for single-frequency and narrowband propagation, respectively. Because the AET experiment transmitted pulses, the definitions of these parameters are not precise. The $\Lambda$ parameter is ambiguous because the concept of a Fresnel zone is inherently single-frequency, and the expression for $\tau_{0}$ assumes small frequency separations. One of the main issues here centers on the question of the spatial scales of the sound field. For CW transmission, the logical acoustic scale of a ray path is described by the Fresnel zone, while for broad-band transmissions it is the ray-tube which is significant [14].

1) A Simple Model of Ray-Tubes The subject of ray-tubes has been rigorously treated by Bowlin [14] for an arbitrary sound-speed profile, but a simple model of ray-tubes can be constructed for a constant sound-speed channel $\left(c=c_{0}\right)$. The total acoustic field can be written

$$
\begin{aligned}
& \Psi(x, z, t) \\
& \quad=\operatorname{Re}\left[\sum_{j=1}^{j=\infty} \int_{\sigma_{0}}^{\sigma_{f}} d \sigma P(\sigma) B_{j}\left(z_{s}\right) B_{j}\left(z_{r}\right) \exp \left(i\left(k_{x} x-\sigma t\right)\right)\right]
\end{aligned}
$$



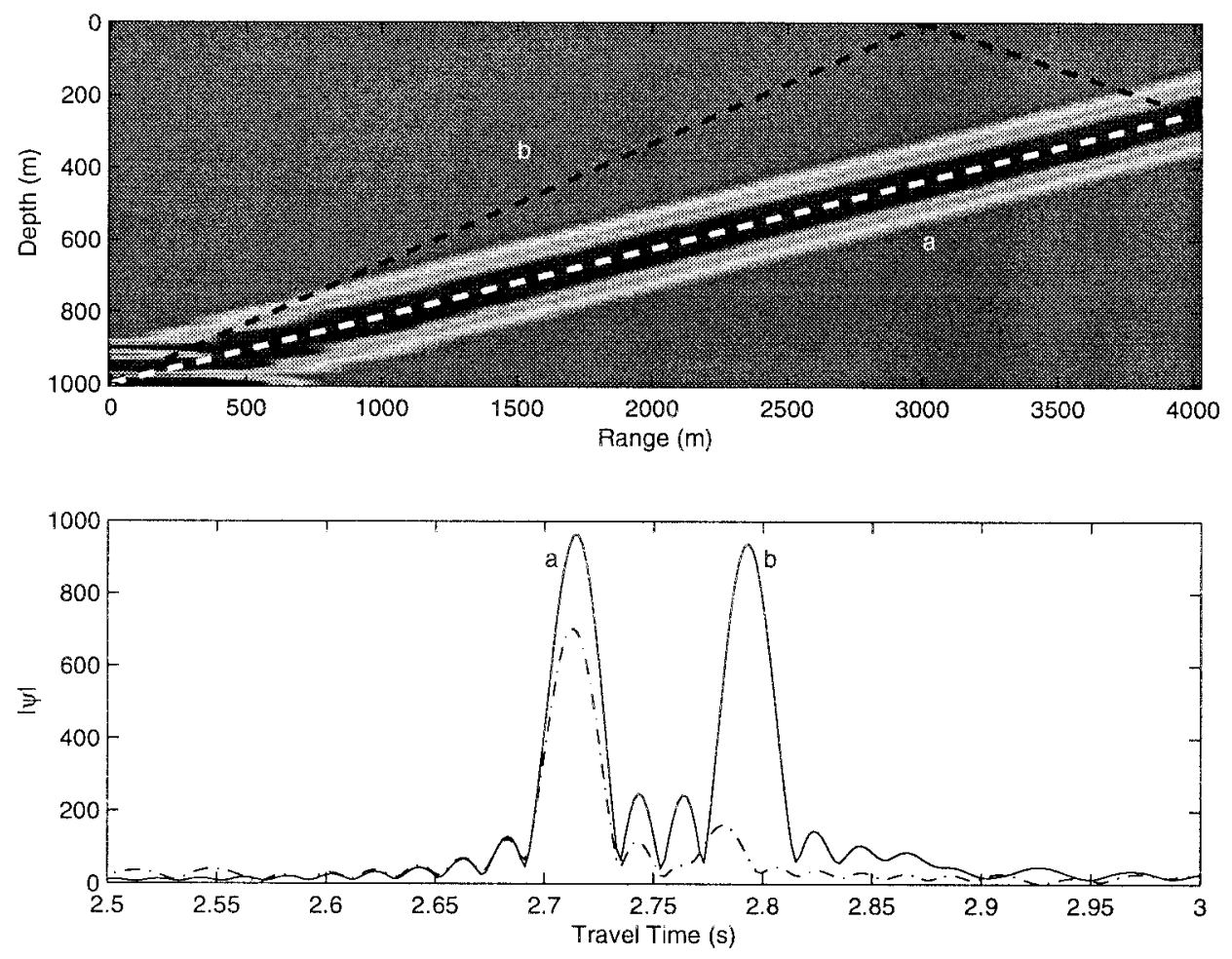

Fig. 3. Upper panel: ray tube width for ray path (a) for a constant sound-speed channel $\left(c_{0}=1500 \mathrm{~m} / \mathrm{s}\right)$. Gray scale is linear in amplitude and ray paths ( $\mathrm{a}$ and $\mathrm{b}$, dashed) are shown for reference. The ray tube is calculated using a partial sum over acoustic normal modes which are within $\pm 7 \mathrm{~m} / \mathrm{s}$ of the group velocity of ray (a) (1474/s). Lower panel: time arrival pattern for two rays (a) and (b) for a receiver at 250-m depth and 4000-m range calculated by summing over all of the acoustic normal modes (solid line) and the partial sum for ray (a) (dash-dot line).

where $P$ is the source spectrum, the vertical modes are $B_{j}(z)=\sin \left(k_{z} z\right), k_{z}=\pi j / z_{b}$ from the boundary condition, and the dispersion relation is $\sigma=c_{0}\left(k_{x}^{2}+k_{z}^{2}\right)^{1 / 2}$. The modal group velocity is $v_{g_{x}}=k_{x} c_{0} /\left(k_{x}^{2}+k_{z}^{2}\right)^{1 / 2}$. The ray-tube problem can be approached when it is realized that a ray is a superposition of acoustic normal modes which have group velocities very close to the group velocity of the ray [21], [22]. Therefore, to form a ray from (10), the summation is restricted to modes which are within some small neighborhood of a chosen group velocity; call this velocity $v_{g 0}$. To calculate a ray path, (10) is modified as follows:

$$
\begin{gathered}
\Psi(x, z)=\operatorname{Re}\left[\sum_{-\delta<v_{g}(\sigma, j)-v_{g 0}<\delta} P(\sigma) B_{j}\left(z_{s}\right) B_{j}\left(z_{r}\right)\right. \\
\left.\quad \times \exp \left(i x\left(k_{x}-\sigma / v_{g 0}\right)\right)\right]
\end{gathered}
$$

where time has been replaced in the equation with $x / v_{g 0}$.

As an example, take: $c_{0}=1500 \mathrm{~m} / \mathrm{s}, z_{b}=2000 \mathrm{~m}$, $v_{g 0}=1474 \mathrm{~m} / \mathrm{s}, P$ a cosine window, $\delta=7 \mathrm{~m} / \mathrm{s}, \sigma_{0}=50$ $\mathrm{Hz}$, and $\sigma_{f}=100 \mathrm{~Hz}$ (50-Hz bandwidth). Fig. 3 shows the ray tube for the eigenray which travels in a straight line and is received at a range of $4000 \mathrm{~m}$ and a depth of $250 \mathrm{~m}$. Fig. 3 also shows the time arrival pattern at the receiver for the total field and the restricted mode sum. The arrival (a) is well modeled by the restricted mode sum. The ray-tube width is constant along the ray, and its width at half maximum is closely given by $\xi=c_{0} / \alpha=30 \mathrm{~m}$. In contrast, the Fresnel radius for this ray is given by

$$
R_{f}^{2}=\lambda \frac{s(R-s)}{R}
$$

where $\lambda$ is the acoustic wavelength, $s$ is the distance along the ray, and $R$ is the total length of the ray. The Fresnel radius is zero at the source and receiver and reaches a maximum value midway along the ray of $140 \mathrm{~m}$. Clearly, our phenomenological description of the ray-tube width $\xi$ does not reduce to the Fresnel radius in the limit of $\mathrm{CW}$ transmission. This model is presented here to demonstrate the significance of broad-band effects.

The agreement between the simple expression $\xi=c_{0} / \alpha$ and this constant sound-speed model, and the rough agreement of our model with the calculations of Bowlin [14], suggest that the ray tube can be treated qualitatively and that a broad-band $\Lambda$ parameter can be defined as follows.

The diffraction parameter $\Lambda$ is calculated in two ways. The standard calculation is for the $\mathrm{CW}$ case, $\Lambda_{\mathrm{Cw}}$, and is defined using the first Fresnel-zone radius $R_{f}$ at the center frequency $\sigma_{0}$, as in (3)

$$
\Lambda_{\mathrm{CW}}=\left(1 / \tau^{2}\right) \int_{\Gamma_{0}} d s_{1} \frac{\left\langle\mu^{2}\right\rangle L_{p}}{c^{2}}\left(R_{f} / L_{z}\right)^{2} /(2 \pi)
$$

where $\tau^{2}=\Phi^{2} / \sigma_{0}^{2}$ is the travel-time variance, $R_{f}$ is defined as in (3), and $L_{z}^{-2}=\left\{k_{v}^{2}\right\}$ is defined by

$$
L_{z}=\frac{1}{\rho_{0}} \int_{0}^{\infty} \rho(\Delta z) d \Delta z .
$$




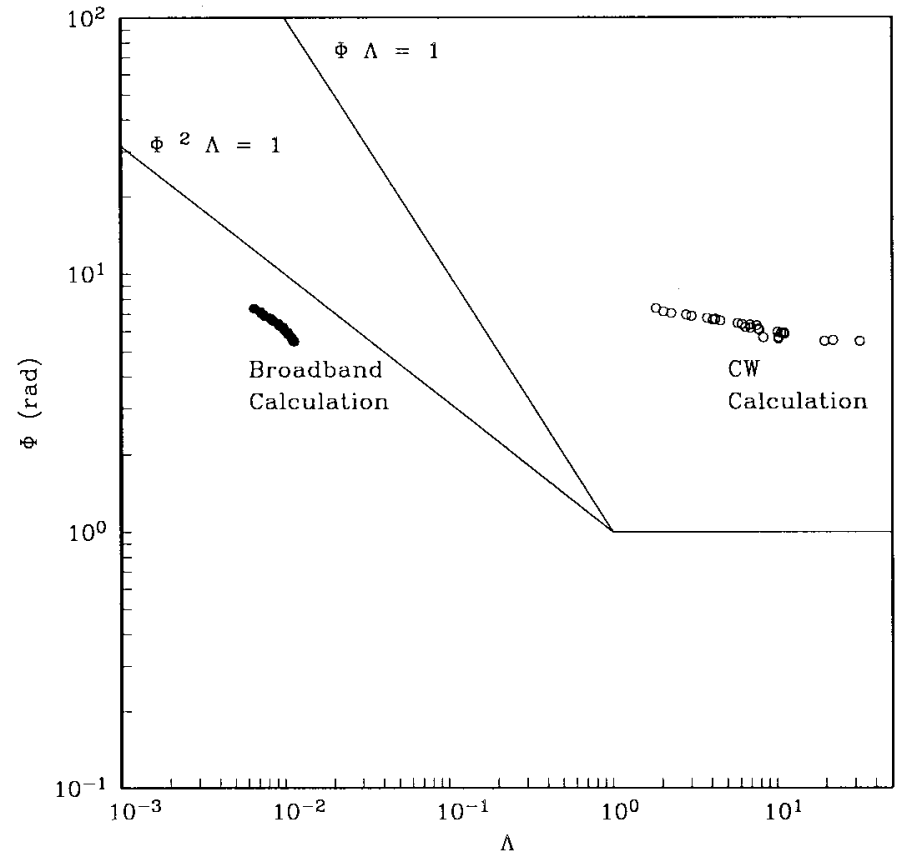

Fig. 4. Lambda Phi ( $\Lambda$ versus $\Phi)$ diagram for the AET resolved wavefronts.

For a broad-band case, the Fresnel radius $R_{f}$ is replaced with a constant ray bundle width $\xi=c_{0} / \alpha=40 \mathrm{~m}(\alpha=37.5 \mathrm{~Hz}$ is the bandwidth of the AET signals), yielding the estimate for $\Lambda_{\mathrm{bb}}$

$$
\Lambda_{\mathrm{bb}}=\left(1 / \tau^{2}\right) \int_{\Gamma_{0}} d s_{1} \frac{\left\langle\mu^{2}\right\rangle L_{p}}{c^{2}}\left(\xi \sec \theta / L_{z}\right)^{2} /(2 \pi) .
$$

Fig. 4 shows our $\mathrm{CW}$ and broad-band calculations for $\Lambda$ for the $\Lambda-\Phi$ diagram for the AET experiment. The calculations for this $\Lambda-\Phi$ diagram are done as described by Colosi $e t$ al. [13]. The $\Lambda_{\mathrm{bb}}$ values are about a factor of 1000 smaller than the values of $\Lambda_{\mathrm{cw}}$. The $\Lambda_{\mathrm{bb}}$ value can be understood qualitatively in terms of the value of $L_{z}$ at the upper turning points of the rays. Taking an average value of $L_{z}$ to be about $130 \mathrm{~m}$, then $(2 \pi)^{-1}\left(\xi / L_{z}\right)^{2}=0.015$, which is a typical value of $\Lambda_{\mathrm{bb}}$ in Fig. 4. For the CW case, the $\Lambda_{\mathrm{cw}}$ values are much greater than 1 . This can be understood qualitatively in terms of the constant-sound-speed Fresnel zone [see (12)] where $s=R / 2, R=3000 \mathrm{~km}$, and $\lambda=20 \mathrm{~m}$ gives $R_{f}=3870 \mathrm{~m}$. When there is a sound channel, the envelope of $R_{f}^{2}$ is close to the parabolic shape in (12), but $R_{f}^{2}$ oscillates between zero at the ray turning points and its maximum value somewhere in between [13]. These values of $R_{f}$ which are close to the scale of the sound channel are clearly unreasonable, since they predict that the sound field would be insensitive to even the sound channel.

Again, it is reiterated that our broad-band calculation of $\Lambda$ is not physics-based and is not related in any way to the statistics of the acoustic field of pulses. Rather, it is a measure of the average scales of the pulse wavefield to the vertical scales of the internal wave field, with the understanding that the physical effect of diffraction is determined by this ratio.

In considering the ray-tube calculation, it must be understood that internal waves can dramatically modify the ray tube, and therefore even Bowlin's method has limitations. Work by Simmen et al. [10] has shown that internal waves can significantly expand the ray tube due to micromultipath generation. A complete description of the ray tube as a function of internal wave field parameters and the mean sound-speed profile is a fundamental unsolved problem in ocean acoustic WPRM.

2) Born Approximation for Pulses: The broad-band calculation of the diffraction parameter is compelling, but a more physical understanding is sought to describe the effects of pulse propagation through a random media. Another simple model starts with the wave equation

$$
\nabla^{2} \psi+k^{2} \psi=2 k^{2} \mu \psi
$$

for constant sound speed $c_{0}$, fractional sound-speed perturbation $\mu=\delta c / c_{0}$, and wavenumber $k=\sigma / c_{0}$ where $\sigma$ is the wave frequency. The first Born approximation to (16) is written as

$$
\psi(\mathbf{r}, \sigma)=\psi_{0}(\mathbf{r}, \sigma)-2 k^{2} \int_{V^{\prime}} G\left(\mathbf{r}-\mathbf{r}^{\prime}, \sigma\right) \mu\left(\mathbf{r}^{\prime}\right) \psi_{0}\left(\mathbf{r}^{\prime}, \sigma\right) d^{3} \mathbf{r}^{\prime}
$$

where $G\left(\mathbf{r}-\mathbf{r}^{\prime}, \sigma\right)=\exp \left(i k\left|\mathbf{r}-\mathbf{r}^{\prime}\right|\right) /\left(4 \pi\left|\mathbf{r}-\mathbf{r}^{\prime}\right|\right)$ is the spherical Greens function. For small angle scattering from a point source $\psi_{0}=\exp (i \mathbf{k} \cdot \mathbf{r}) /|\mathbf{r}|$ and the observation position at $\mathrm{r}=(R, 0,0)$, the wavefunction becomes

$$
\begin{aligned}
\psi(\mathbf{r}, \sigma)= & \psi_{0}(\mathbf{r}, \sigma)\left[1-\frac{k^{2}}{2 \pi} \int_{0}^{R} d x^{\prime} \frac{R}{x^{\prime}\left(R-x^{\prime}\right)} \int_{-\infty}^{\infty} d y^{\prime}\right. \\
& \left.\times \int_{-\infty}^{\infty} d z^{\prime} \mu\left(\mathbf{r}^{\prime}\right) \exp \left(i k \frac{R\left(y^{\prime 2}+z^{\prime 2}\right)}{2 x^{\prime}\left(R-x^{\prime}\right)}\right)\right] \cdot
\end{aligned}
$$

Next, a pulse is formed using the Fourier transform so

$$
\Psi(\mathbf{r}, t)=\int_{-\infty}^{\infty} P(\sigma) \psi(\mathbf{r}, \sigma) e^{-i \sigma t} d \sigma
$$

where $P(\sigma)=\left(1 / \sqrt{2 \pi \alpha^{2}}\right) \exp \left(-\left(\sigma-\sigma_{0}\right)^{2} /\left(2 \alpha^{2}\right)\right)$ is a Gaussian source transfer function. The frequency integral can be done analytically, yielding

$$
\Psi(\mathrm{r}, t)=\Psi_{0}(\mathbf{r}, t)\left(1+\varphi_{1}\right)
$$

where

$$
\Psi_{0}(\mathbf{r}, t)=\exp (i \delta \theta) \exp \left(-q^{2} \delta \theta^{2} / 2\right) / R
$$

and

$$
\begin{aligned}
\varphi_{1}(\mathrm{r}, t)= & -k_{0} \int_{0}^{R} d x^{\prime} \int_{-\infty}^{\infty} d Y \int_{-\infty}^{\infty} d Z \mu\left(x^{\prime}, R_{f} Y, R_{f} Z\right) \\
& \times \exp \left(i \delta \phi-\frac{q^{2}}{2}\left(\delta \phi^{2}+2 \delta \phi \delta \theta\right)\right) \\
& \times\left[1+q^{2}\left(1-q^{2}(\delta \theta+\delta \phi)^{2}+2 i(\delta \theta+\delta \phi)\right)\right]
\end{aligned}
$$

with

$$
\delta \phi=\pi\left(Y^{2}+Z^{2}\right) .
$$




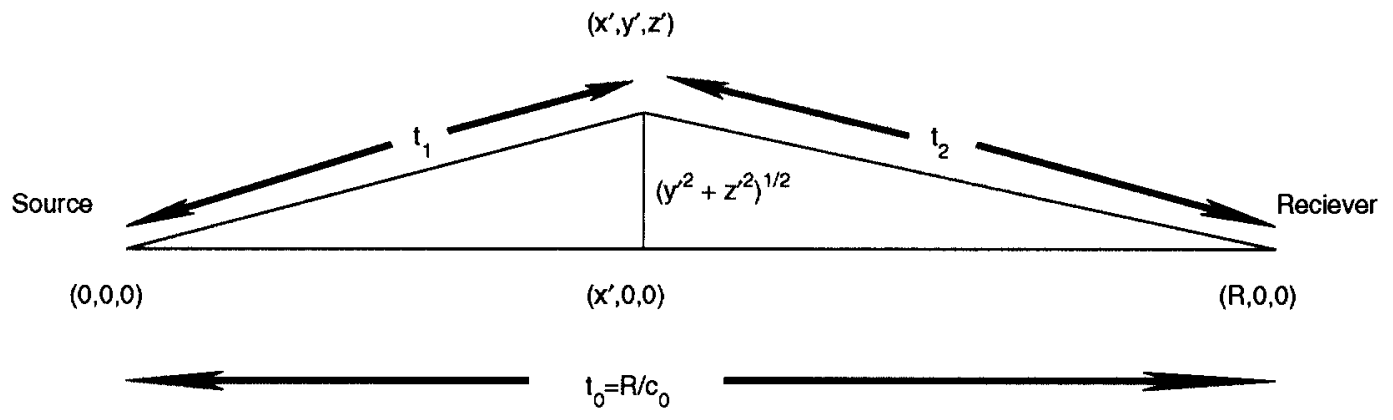

Fig. 5. Geometry of the broad-band Born approximation problem.

Here $\delta \theta=\sigma_{0}\left(R / c_{0}-t\right), q=\alpha / \sigma_{0}, k_{0}=\sigma_{0} / c_{0}$, and variables have been changed from $\left(y^{\prime}, z^{\prime}\right)$ to $Y=y^{\prime} / R_{f}\left(x^{\prime}\right)$ and $Z=z^{\prime} / R_{f}\left(x^{\prime}\right)$. Here $R_{f}$ is the first Fresnel-zone radius given by $R_{f}^{2}=\lambda_{0} x^{\prime}\left(R-x^{\prime}\right) / R$, and $\lambda_{0}$ is the wavelength at the center frequency. Geometrically, $\delta \phi$ is the center-frequency phase difference between the straight-line path to the receiver and the path scattered from point $\left(x^{\prime}, y^{\prime}, z^{\prime}\right)$ (see Fig. 5). For $q=0$, the standard single-wave Born approximation is recovered [i.e., the second term in (18)].

From (20) and the condition that $\varphi_{1} \ll 1$, it is seen that $\Psi(\mathrm{r}, t) \simeq \Psi_{0}(\mathbf{r}, t) \exp \left(\varphi_{1}\right) \cdot \operatorname{Re}\left(\varphi_{1}\right)$ is, therefore, associated with variations in the log-amplitude $(\chi)$ of the signal and $\operatorname{Im}\left(\varphi_{1}\right)$ is associated with variations in the signal phase $(\phi)$.

It is helpful to examine the spatial weighting properties for $\chi$ and $\phi$. Equation (22) can be rewritten as

$$
\begin{array}{r}
\varphi_{1}(\mathrm{r}, t)=-k_{0} \int_{0}^{R} d x^{\prime} \int_{-\infty}^{\infty} d Y \int_{-\infty}^{\infty} d Z \mu\left(x^{\prime}, R_{f} Y, R_{f} Z\right) \\
\times\left[W_{\chi}+i W_{\phi}\right]
\end{array}
$$

where

$$
\begin{aligned}
W_{\chi}= & W_{e}(\delta \phi, \delta \theta, q)\left[W_{\chi_{1}}\left(1+q^{2}-q^{4}(\delta \theta+\delta \phi)^{2}\right)\right. \\
& \left.-W_{\phi_{1}}\left(2 q^{2}(\delta \phi+\delta \theta)\right)\right] \\
W_{\phi}= & W_{e}(\delta \phi, \delta \theta, q)\left[W_{\chi_{1}}\left(2 q^{2}(\delta \theta+\delta \phi)\right)\right. \\
& \left.+W_{\phi_{1}}\left(1+q^{2}-q^{4}(\delta \theta+\delta \phi)^{2}\right)\right]
\end{aligned}
$$

and

$$
W_{e}=\exp \left(-\frac{q^{2}}{2}\left(\delta \phi^{2}+2 \delta \phi \delta \theta\right)\right) .
$$

Here the single-wave results (i.e., $q=0$ ) are

$$
\begin{aligned}
& W_{\chi_{1}}=\cos (\delta \phi) \\
& W_{\phi_{1}}=\sin (\delta \phi) .
\end{aligned}
$$

The variances of log-amplitude and phase can now be compactly written as

$$
\begin{aligned}
\left\langle\chi^{2}\right\rangle=k_{0}^{2} \int_{0}^{R} d x_{1} \int_{-\infty}^{\infty} d Y_{1} \int_{-\infty}^{\infty} d Z_{1} \int_{0}^{R} d x_{2} \int_{-\infty}^{\infty} d Y_{2} \\
\times \int_{-\infty}^{\infty} d Z_{2}\left\langle\mu\left(\mathbf{r}_{1}\right) \mu\left(\mathbf{r}_{2}\right)\right\rangle W_{\chi}\left(\mathbf{r}_{1}\right) W_{\chi}\left(\mathbf{r}_{2}\right)
\end{aligned}
$$

and

$$
\begin{aligned}
\left\langle\phi^{2}\right\rangle= & k_{0}^{2} \int_{0}^{R} d x_{1} \int_{-\infty}^{\infty} d Y_{1} \int_{-\infty}^{\infty} d Z_{1} \int_{0}^{R} d x_{2} \int_{-\infty}^{\infty} d Y_{2} \\
& \times \int_{-\infty}^{\infty} d Z_{2}\left\langle\mu\left(\mathbf{r}_{1}\right) \mu\left(\mathbf{r}_{2}\right)\right\rangle W_{\phi}\left(\mathbf{r}_{1}\right) W_{\phi}\left(\mathbf{r}_{2}\right)
\end{aligned}
$$

where $\mathbf{r}_{\mathbf{1}}=\left(x_{1}, Y_{1}, Z_{1}\right)$ and $\mathbf{r}_{\mathbf{2}}=\left(x_{2}, Y_{2}, Z_{2}\right)$. The weighting functions $W_{\chi}\left(\mathbf{r}_{1}\right) W_{\chi}\left(\mathbf{r}_{\mathbf{2}}\right)$ and $W_{\phi}\left(\mathbf{r}_{\mathbf{1}}\right) W_{\phi}\left(\mathbf{r}_{\mathbf{2}}\right)$ tell us the spatial weighting on the correlation function of $\mu$.

Here the effects of the signal bandwidth can be seen, and the physically significant factor is $q$, the bandwidth over the center frequency. First, nonzero $q$ introduces an exponential modulation term depending on $q^{2}$ [see (27)], which confines the influence of $\mu$ to a volume closer to the direct path of the wavepacket (i.e., the $x$ axis). This modulation term also depends on the phase delay $\delta \theta$. For $\delta \theta>0$, which corresponds to early arriving energy on the rise of the pulse, there is strong confinement along the $x$ axis. However, for $\delta \theta<0$, which corresponds to late arriving energy on the fall of the pulse, the modulation term becomes exponentially increasing until some distance away from the $x$ axis that $\delta \phi>2 \delta \theta$ where exponential decrease takes place. Physically, this effect for $\delta \theta<0$ is the influence of scattered energy away from the $x$ axis but, because of the longer path length, takes longer to get to the receiver (see Fig. 5).

Furthermore, the $q$ factor introduced a complex amplitude term [square brackets in (22)] which mixes the single-wave weighting functions $W_{\chi_{1}}$ and $W_{\phi_{1}}$. The magnitude of the mixing depends on $q^{2}$. Also, it is seen from (22) that the Fresnel radius at the center frequency plays a key role in the spatial weighting of $\mu\left(x^{\prime}, Y, Z\right)$. In contrast to the ray-tube picture which showed that the ray-tube extent was constant along the ray, (22) shows that parts of the ray path are affected differently.

As an example, a case relavant to the ATOC signal is taken with $\sigma_{0}=75 \mathrm{~Hz}, \alpha=45.2 \mathrm{~Hz}$ (3-dB point at 37.5 $\mathrm{Hz} ; q=0.6)$ [6], and $c_{0}=1500 \mathrm{~m} / \mathrm{s}$. Figs. 6-8 show the weighting functions for our test case and for $\tau=\delta \theta / \sigma_{0}=$ $\left( \pm\left(2 / \alpha^{2}\right)^{1 / 2}, 0\right)$. The weighting functions are shown for $\mathbf{r}_{1}=$ $\mathrm{r}_{2}$, therefore $W_{\chi}^{2}, W_{\phi}^{2}, W_{\chi_{1}}^{2}$, and $W_{\phi_{1}}^{2}$ are plotted.

In Fig. 6, for $\tau=0$, one can see the effect of the exponential modulation term [see (27)], and the pulse weighting function decays much more rapidly than the $\mathrm{CW}$ case. Here the effect of the mixing of $W_{\chi_{1}}$ and $W_{\phi_{1}}$ is small for small values of $\left(Y^{2}+Z^{2}\right)^{1 / 2}$ because $\delta \theta \simeq 0$. 
(a)

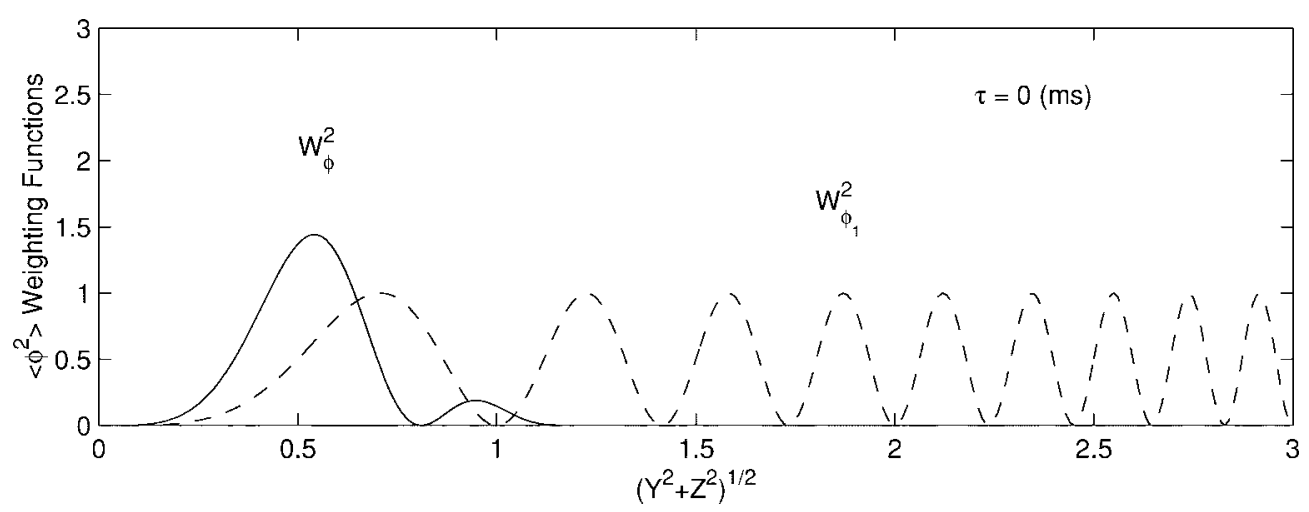

(b)

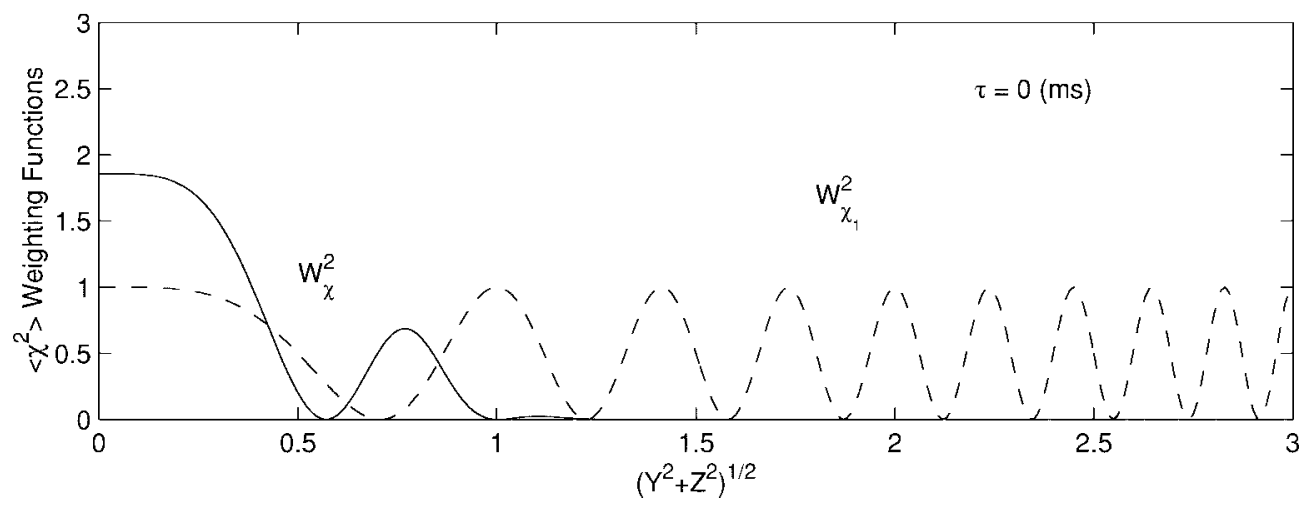

Fig. 6. Example weighting functions for (a) $\left\langle\phi^{2}\right\rangle$ and (b) $\left\langle\chi^{2}\right\rangle$ with $\tau=0 \mathrm{~ms}$.

(a)

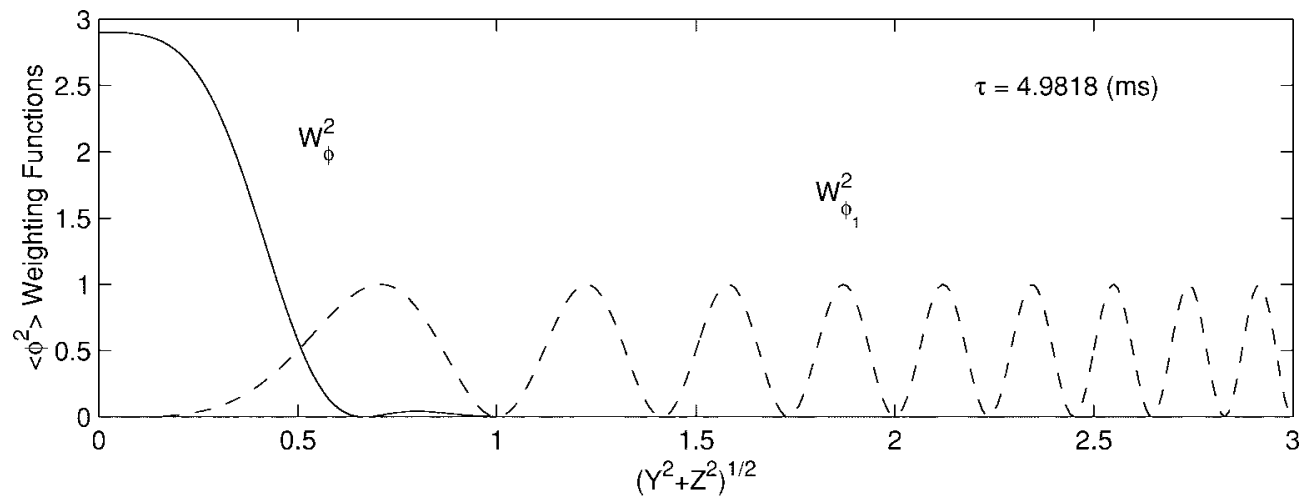

(b)

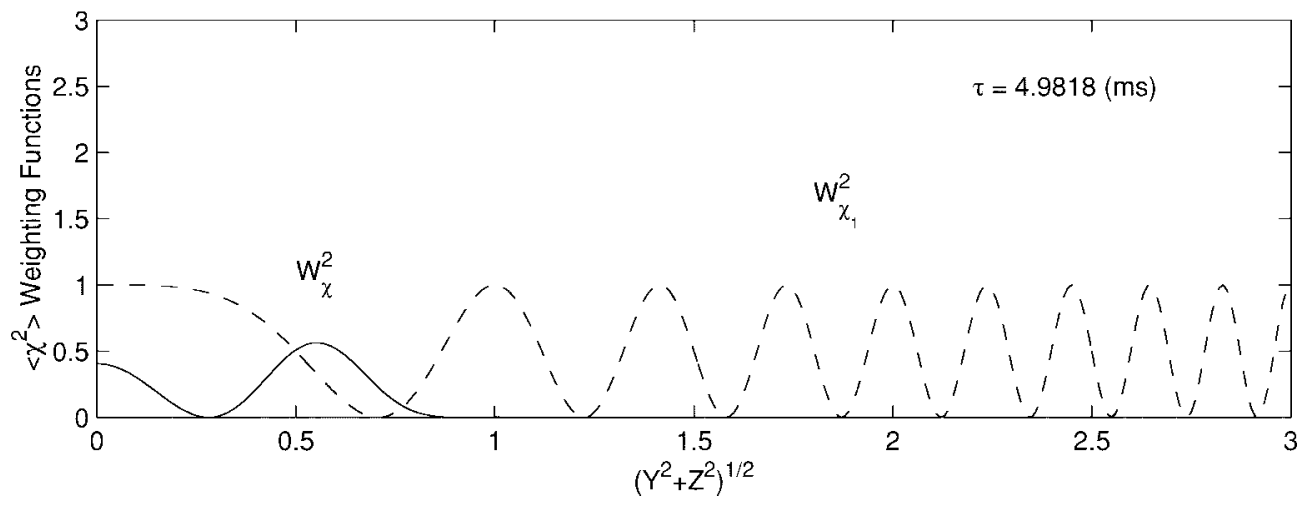

Fig. 7. Example weighting functions for (a) $\left\langle\phi^{2}\right\rangle$ and (b) $\left\langle\chi^{2}\right\rangle$ with $\tau=\delta \theta / \sigma_{0}=\left(2 / \alpha^{2}\right)^{1 / 2}=4.9818 \mathrm{~ms}$.

In Fig. 7, for $\tau=+4.9 \mathrm{~ms}$, which corresponds to the rise of the unperturbed pulse, the mixing of the weighting functions is clearly evident; $W_{\phi}^{2}$ does not start at zero. Also, the exponential modulation term strongly affects the weighting functions, and the weighting is closer to the $x$ axis than in the $\tau=0$ case. 
(a)

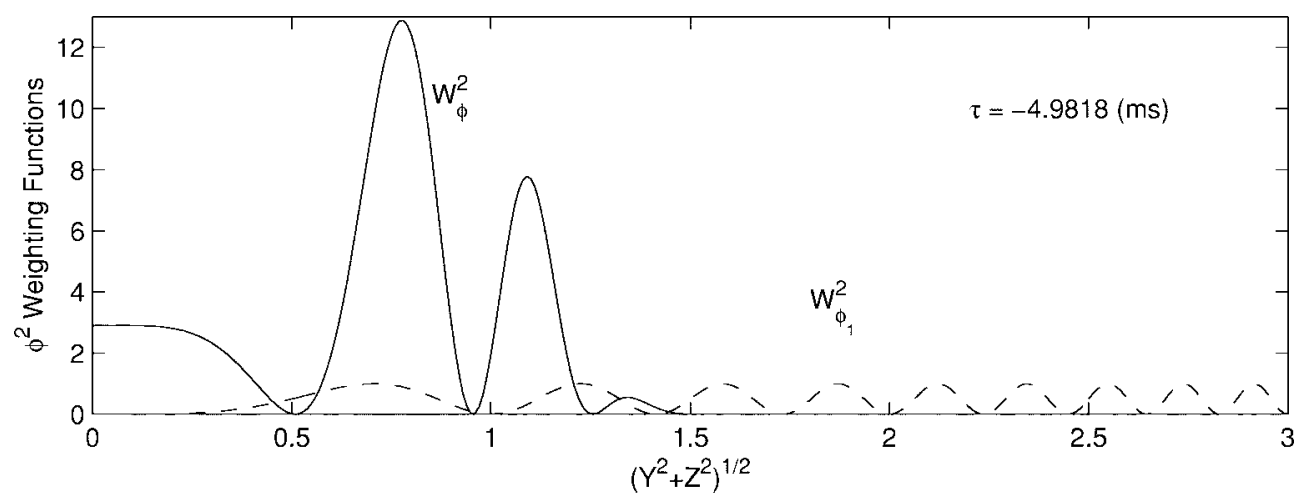

(b)

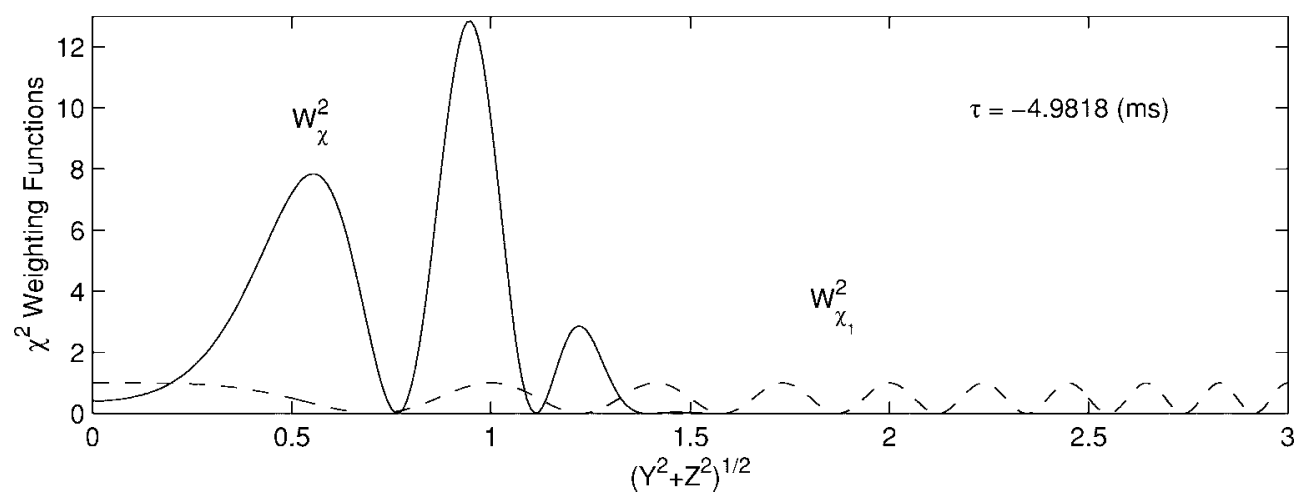

Fig. 8. Example weighting functions for $\left\langle\chi^{2}\right\rangle$ and $\left\langle\phi^{2}\right\rangle$ with $\tau=-4.9818 \mathrm{~ms}$.

Finally in Fig. 8, for $\tau=-4.9 \mathrm{~ms}$, which corresponds to the fall of the unperturbed pulse, the weighting functions are further away from the $x$ axis. This effect occurs because this late arriving energy must scatter from points further away from the $x$ axis.

It is clear from Figs. 6-8 that pulse scattering is very different from the $\mathrm{CW}$ case. The broad-band weighting functions essentially place a cutoff on the sound-speed fluctuation correlation function at the first Fresnel-zone radius whereas the $\mathrm{CW}$ weighting functions impose no such cutoff; that is, pulse propagation is insensitive to scales larger than $R_{f}$. At the same time, it is clear that the ray-tube ideas presented in the previous section are a gross oversimplification because $\chi$ and $\phi$ have different sensitivities to the random medium, and different parts of the propagation path are all not equally affected by the random medium; that is, there is some effective broad-band Fresnel radius which clearly de-emphasizes the end-points of the propagation path and emphasizes the middle, as can be seen from (22). The broad-band effect shown here, however, is not a factor of 100 or 1000 effect, as would be required to describe the AET results. This suggests that more work is needed to understand the AET results.

\section{E. Discussion}

It has been shown that the value of $\Lambda$ is quite sensitive to the treatment of the broad-band character of the pulse; $\Lambda_{\mathrm{Cw}}$ and $\Lambda_{\mathrm{bb}}$ differ by a factor of 1000 ! Therefore, the wave propgation regime is discussed under the assumption that the $\Lambda_{\mathrm{bb}}$ calculation that has been done is at least roughly appropriate. Thus, the experiment is predicted to behave as though it were near the boundary of the weak-fluctuation and strong-fluctuation regimes.

The observations of $\sigma_{\ln I}^{2}$ in the AET experiment are between 0.21 and 0.51 [13]; these are at the limit of and in excess of weak-fluctuation values. Since the PDF's are close to lognormal rather than exponential, the transmissions appear to be not far from the weak-fluctuation regime, if analogies with optical propagation through turbulence are any guide [23].

The observed pulse spread $\tau_{0}$ is smaller than the rms traveltime variance by a factor of 3 . This is again consistent with unsaturated or partially saturated behavior.

The small spread results from the AET have important implications for large-scale tomography, since pulse spread and internal wave bias are closely related [see (9)]. The small observed spread of 1 to $5 \mathrm{~ms}$ implies an equally small internal wave bias.

A theory of pulse propagation is needed to make accurate estimates of wave-propagation regime (through $\Lambda$ ), pulse spread, and PDF. The simple Born approximation results presented here are a good starting place. For the future, it is desirable to have simpler analytical expressions for $\left\langle\chi^{2}\right\rangle$ and $\left\langle\phi^{2}\right\rangle$ than (30) and (31) give, and it is necessary to account for a general waveguide. However, there are clear range limitations to the applicability of the Born approximation which is a single scatter theory, and therefore the arrival of a fully broad-band theory for long-range basin-scale transmissions might be very far away.

Thus, we have come full circle since the SLICE89 experiment. After the SLICE89 analysis, it was discovered that acoustic scattering in the pulse cresendo was much stronger 
(a)

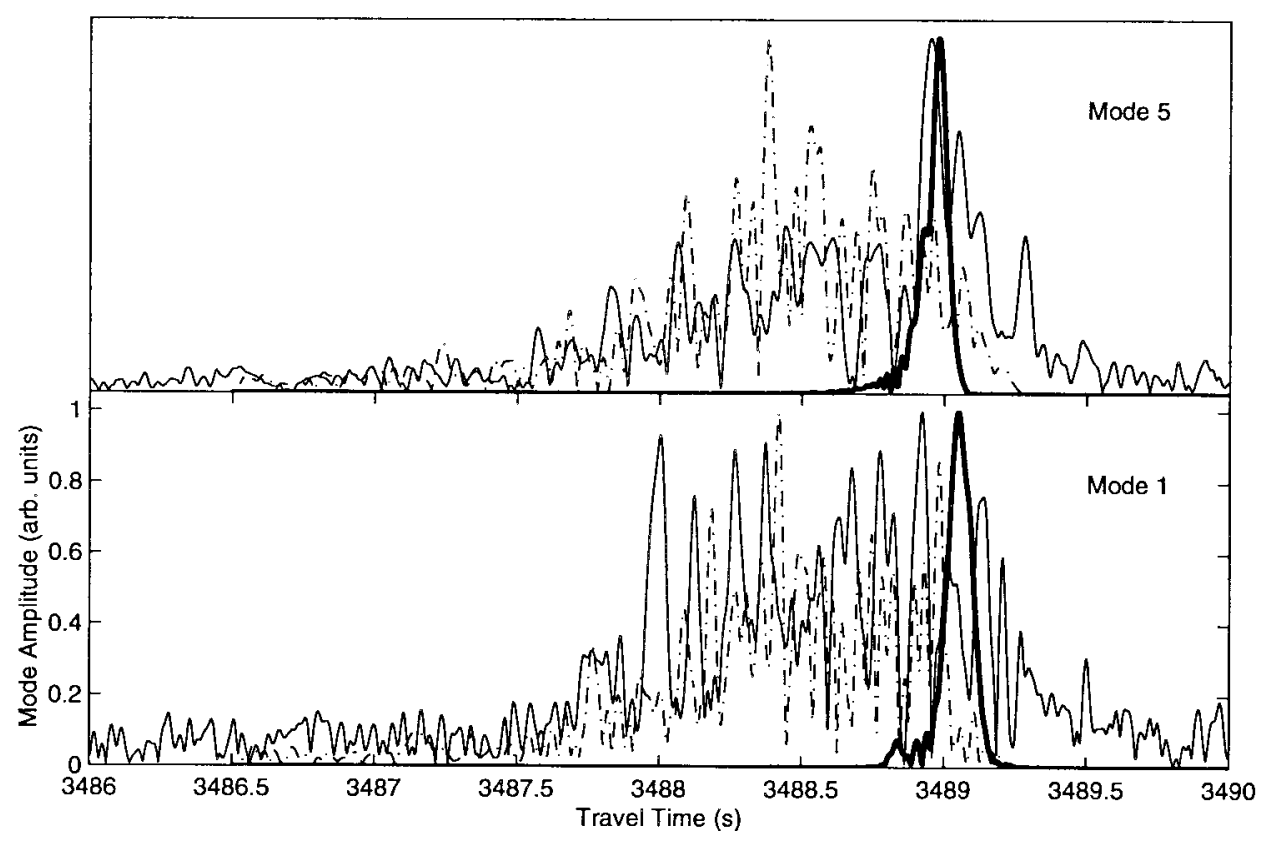

Fig. 9. The arrival patterns of (a) mode 5 and (b) mode 1 from the Pioneer Seamount source as observed at the ATOC VLA2 at Kiritimati (light line). Simulated mode arrivals with (dash-dot line) and without (dark line) GM internal waves.

than anticipated. Now, after analysis of the ATOC AET data, it is seen that the early ray-like arrivals show much weaker scattering than previously thought.

\section{ACOUSTICAL OCEANOGRAPHY}

Acoustic transmission data can be used to interpret oceanographic variability, and, therefore, random media effects are important to understand. For monitoring of large-scale ocean heat content or mesoscale eddies, internal waves impose the ultimate resolution limits much as the resolution limits of ground-based telescopes are imposed by atmospheric turbulence. For monitoring of small-scale ocean processes like internal waves, acoustic observables are sought which show large effects because detectability is often an issue. Also, the functional relationship between the parameters of an internal wave model and a set of acoustic observables must be known.

\section{A. Acoustic Thermometry}

Basin-scale acoustic thermometry has been undertaken by the ATOC program to test large-scale climate models. As a measurement technique, it complements satellite remote sensing of the ocean surface [24]. The promise of acoustic thermometry is to provide vertical resolution of ocean climate variability-resolution that a satellite cannot provide (horizontal resolution for satellites is excellent while for acoustic methods it is poor). It is clear that internal waves are limiting the identification of the ray paths near the sound channel axis and the idenfied rays for the AET are all closely clustered to the ocean surface [6], [13]. This case is similar to SLICE89 [15] (see Fig. 15); the AET VLA is at a range 3.2 times longer than for SLICE89, but the frequency is $1 / 3$ that of the frequency in SLICE89. For acoustic tomography, resolving a large number of wavefronts with different turning depths is how vertical resolution achieved. If wavefronts could be identified further into the highly scattered mode-like region (as would be the case if the data looked like the lower panels of Figs. 2 or 10), then greater vertical resolution could be achieved; therefore, internal waves limit the resolution of raybased acoustic thermometry. To supplement the ray arrivals and enhance vertical resolution, the final arrival of the AET has been interpreted as a mode 1 arrival [6]. Colosi and Flatté [25] showed that the gravest acoustical mode is the most robust to internal wave sound-speed perturbations. However, Fig. 9 shows that the observed mode 1 and mode 5 arrivals at the Kiritimati VLA for the ATOC transmissions from Pioneer Seamount are highly spread in time relative to the simulation without internal waves. The simulation with internal waves shows comparable time spreading to the observations, and comparisons between simulations with and without internal waves indicate a travel-time bias of the order of several hundreds of milliseconds. These observations indicate large mode coupling. Mode arrivals at the Hawaii VLA show comparable but slightly smaller mode coupling effects.

To examine if tomographic vertical resolution can be increased at lower frequencies (i.e., mode coupling significantly reduced), in May of 1995 the ATOC program performed an alternate source test (AST) in which simultaneous $28-\mathrm{Hz}$ and 84-Hz signals were transmitted by a source suspended near the sound channel axis from a ship located close to Pioneer Seamount. Two simulated time fronts for the Hawaii VLA are shown in Fig. 10. The simulations are calculated using range-dependent sound-speed profiles derived from the annual Levitus 1994 database. Internal wave sound-speed fluctuations were calculated using the method of Colosi and Brown [26], but variations in the internal wave field due to changes in the buoyancy frequency profile and the local Coriolis parameter were not taken into account. For the simulations in Fig. 10, 
(a)

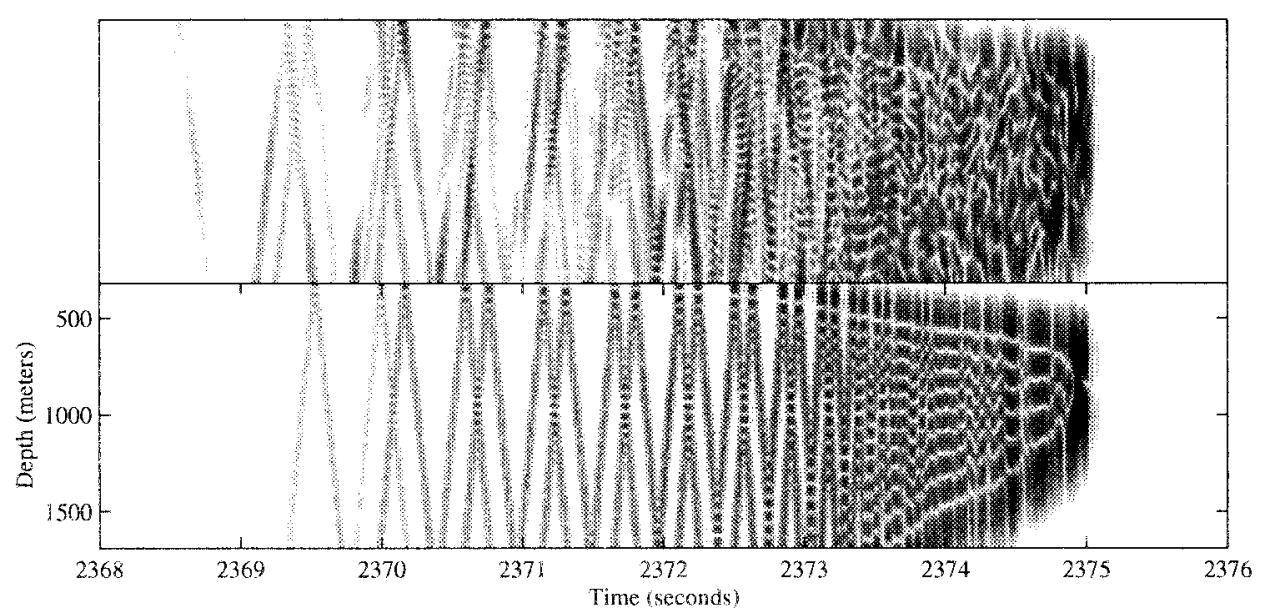

Fig. 10. Time fronts from numerical simulations at a center frequency of $84 \mathrm{~Hz}$ for the ATOC VLA1. (a) Simulation using the Levitus 1994 sound-speed database plus a random realization of internal wave sound-speed perturbations at the GM reference level. (b) Simulation without internal waves.

(a)

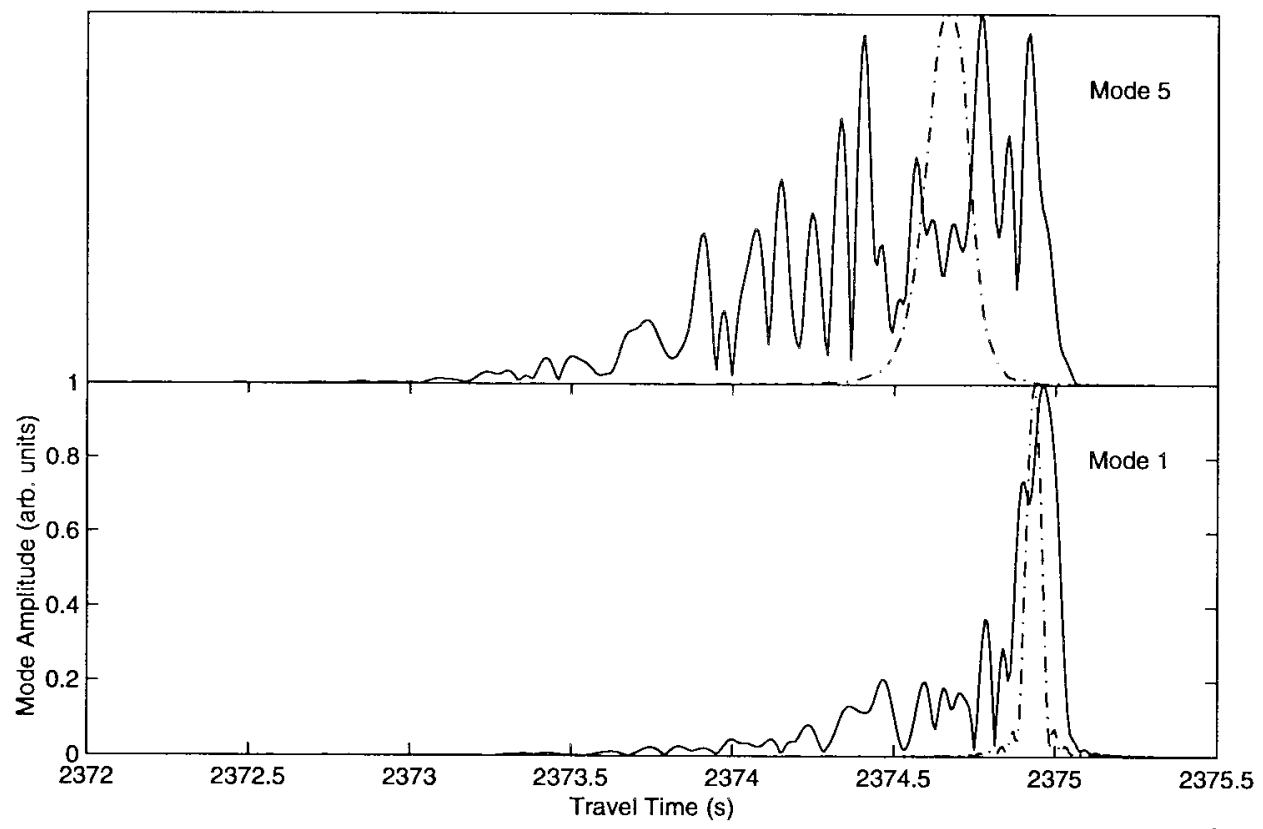

Fig. 11. A realization of the arrival patterns for (a) mode 5 and (b) mode 1 at VLA1 computed from the simulation data shown in Fig. 10. The dash-dot/solid lines are simulated arrival patterns without/with internal wave sound-speed perturbations.

the center frequency is $84 \mathrm{~Hz}$ and the $3-\mathrm{dB}$ bandwidth is $15 \mathrm{~Hz}$ (for the ATOC source the center frequency is $75 \mathrm{~Hz}$ and the bandwidth is $37.5 \mathrm{~Hz}$ ). The general character of the arrival pattern has early arriving stable ray-like energy with small travel-time fluctuations and late arriving highly scattered mode-like energy.

Fig. 11 shows simulated mode 1 and mode 5 arrivals for the Hawaii VLA. For mode 1, the simulation without internal waves shows a sharp peak whereas the simulation with internal waves shows some time spread $(\simeq 0.1 \mathrm{~s})$. This time spreading is the result of mode coupling caused by internal waves; mode 1 does not propagate from the source to the receiver in such a way as to maintain its identity. Mode 1 measured at the receiver is a complex superposition of energy which has propagated as other mode numbers having different group velocities. But, for mode propagation in the deep ocean, mode
1 has the smallest group velocity, and, therefore, the last arriving energy in the mode 1 arrival pattern must be energy which has mostly traveled as mode 1 from source to receiver. Following Headrick [27], this point can be called the pseudoadiabatic mode 1 (PAM1) arrival which can be used in an adiabatic acoustic inverse [6]. This PAM1 arrival is not strictly adiabatic, as there is a travel-time bias associated with it [25]. The bias is on the order of $0.1 \mathrm{~s}$.

Use of higher order modes would also increase the vertical resolution of acoustic thermometry. From Fig. 11, it is clear that mode 5 shows dramatic time spreading $(\simeq 1.0 \mathrm{~s})$. A method for doing the acoustic inverse problem in situations of strong random mode coupling or nonadiabaticity remains a difficult research topic.

Simulations of the arrival pattern at the Hawaii VLA for the $28-\mathrm{Hz}$ signal are shown in Fig. 12, which can be directly 
(a)

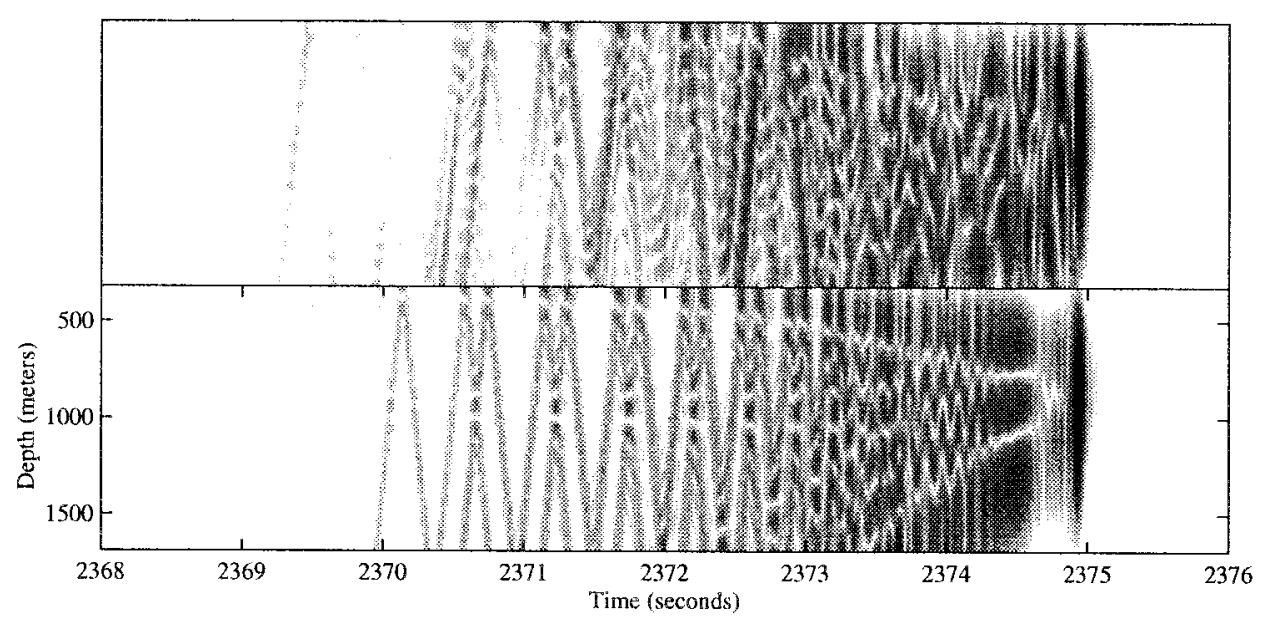

Fig. 12. Time fronts from numerical simulations at a center frequency of $30 \mathrm{~Hz}$ for the ATOC VLA1. (a) A simulation using the Levitus 1994 sound-speed database plus internal wave sound-speed perturbations at the GM reference level. (b) A simulation without internal waves.

(a)

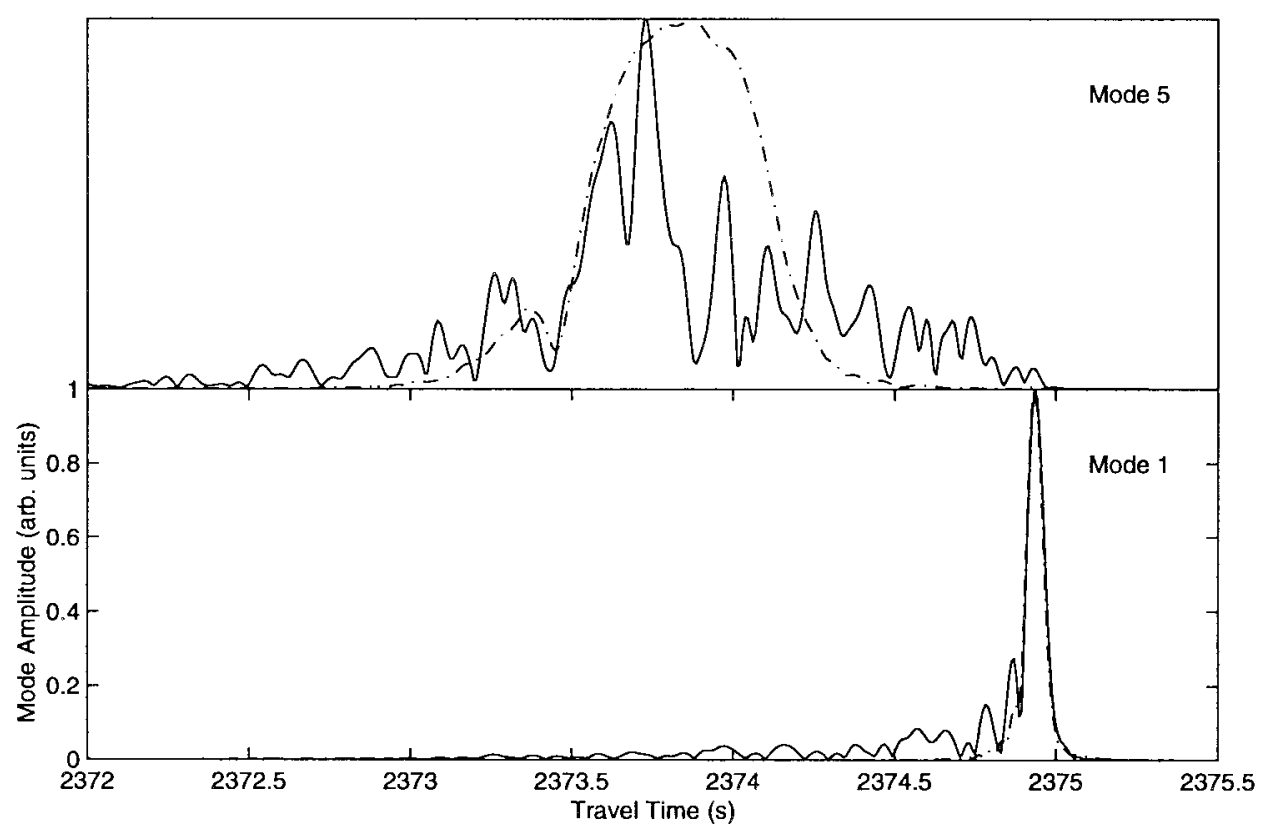

Fig. 13. Arrival patterns for modes (a) 5 and (b) 1 at VLA1 computed from the simulation data shown in Fig. 12. The dash-dot line is a simulated arrival pattern without internal waves and the solid line is a simulated arrival pattern with internal waves.

compared to Fig. 10. The ray arrivals for the low-frequency case are about as clean as the higher frequency case, yielding a similar identification of wavefronts. The biggest difference between the two cases is for the acoustic normal modes.

Fig. 13 shows the arrival patters for modes 1 and 5 at the Hawaii VLA for the $28-\mathrm{Hz}$ signals. Comparing to Fig. 11, it is clear that mode coupling effects are significantly reduced.

The mode 1 arrival is almost completely adiabatic, showing very little time spread. The mode 5 arrival shows fluctuations but the basic envelope of the calculation with internal waves matches the pattern of the no internal wave calculation. Therefore, there is evidence that low-mode arrivals at $28 \mathrm{~Hz}$ are much more robust to internal waves than the $84-\mathrm{Hz}$ case! Whether the AST data shows this dramatic reduction in mode coupling will be revealed in later work.

\section{B. Internal Wave Tomography}

Understanding internal wave generation and dissipation is a first-order oceanographic problem because internal waves serve as an important pathway for the passage of energy from large-scale flows to turbulence and molecular dissipation [28]. Several processes are candidates to supply internal wave energy [29], including wind forcing, instability of mesoscale eddies, and tidal energy conversion. In arguing for the tidal origin of internal waves, Munk states that "the remarkable consistency (within a factor of 2) over a wide range of conditions of both: 1) the internal wave intensity and 2) the diapycnal diffusivity, argues for a more reliable source than just wind energy" [28]. Specific sources of internal waves might be recognized acoustically by the timescale of variability. Tidal 
origin suggests a fortnightly period for acoustic fluctuations, while wind generation suggests a seasonal period due to the development of the winter storm track. Mesoscale sources suggest a geographic variability of acoustic fluctuations, so that acoustic transmission paths which intersect regions of high mesoscale activity, such as the California current system, will have large acoustic fluctuations.

Aspects of the problem of internal wave tomography have been discussed by many researchers [30]-[35]. For short-range transmissions, the Rytov method, which is based on weak fluctuation theory, may prove useful [35], but for long-range transmissions Monte Carlo numerical simulations have been the method of choice. This approach is discussed first for the SLICE89 experiment before continuing on with the issues from the ATOC and AET experiments.

1) SLICE89: The SLICE89 experiment was conducted in the eastern north Pacific over a 10-day period during which narrow pulses were transmitted from a moored $250-\mathrm{Hz}$ center frequency source (source depth $=800 \mathrm{~m}$ ) to a $3-\mathrm{km}$-long vertical array of 50 hydrophones $1000 \mathrm{~km}$ distant. Like the ATOC data, the observed time fronts for SLICE89 showed two distinct regions in the arrival pattern: a highly distorted final arrival, and stable early arriving wavefronts. Due to the sparse vertical array used in SLICE89, individual acoustic modes could not be resolved, so the wavefront envelope is considered here. In the ray-like region, two different measures of wavefront timing fluctuations are used as data for our estimate of internal wave displacements.

The effects of mesoscale sound-speed perturbations on the wavefront envelope are treated first. Fig. 14 shows the wavefront envelope for the SLICE89 data, a simulation using a range-independent sound-speed profile, and a simulation with the range-independent profile plus mesoscale perturbations. The mesoscale perturbation field was determined by objectively mapping environmental data from CTD's, XBT's, and AXBT's [35], [36]. The range-independent sound-speed profile was calculated from the average of 11 CTD casts taken during the experiment (10 CTD's went to 2000-m depth and one went to 4000-m depth). For the SLICE89 data and the numerical simulations with internal waves, the wavefront envelope is defined to be the location in time/depth coordinates of the intensity level which is $2 \%$ of the maximum intensity of the incoherent average of all of the data (110 pulses are averaged for the SLICE89 data and 33 pulses are averaged for the simulation). The calculation of the wavefront envelope for the simulation using the range-independent profile and the mesoscale perturbations used the same reference intensity level as the case with internal waves.

Fig. 14 shows that the observed mesoscale sound-speed field for the SLICE89 experiment has very little effect on the wavefront envelope, and, therefore, the mismatch between the data and the simulations without internal waves is a purely internal wave effect. This result is different from that of Tappert and Spiesberger who showed a significant mesoscale effect for a longer transmission path between Hawaii and northern California [38]. Since the eastern north Pacific is known to be a very quiet region for mesoscale activity, this result may not hold in other parts of the ocean.

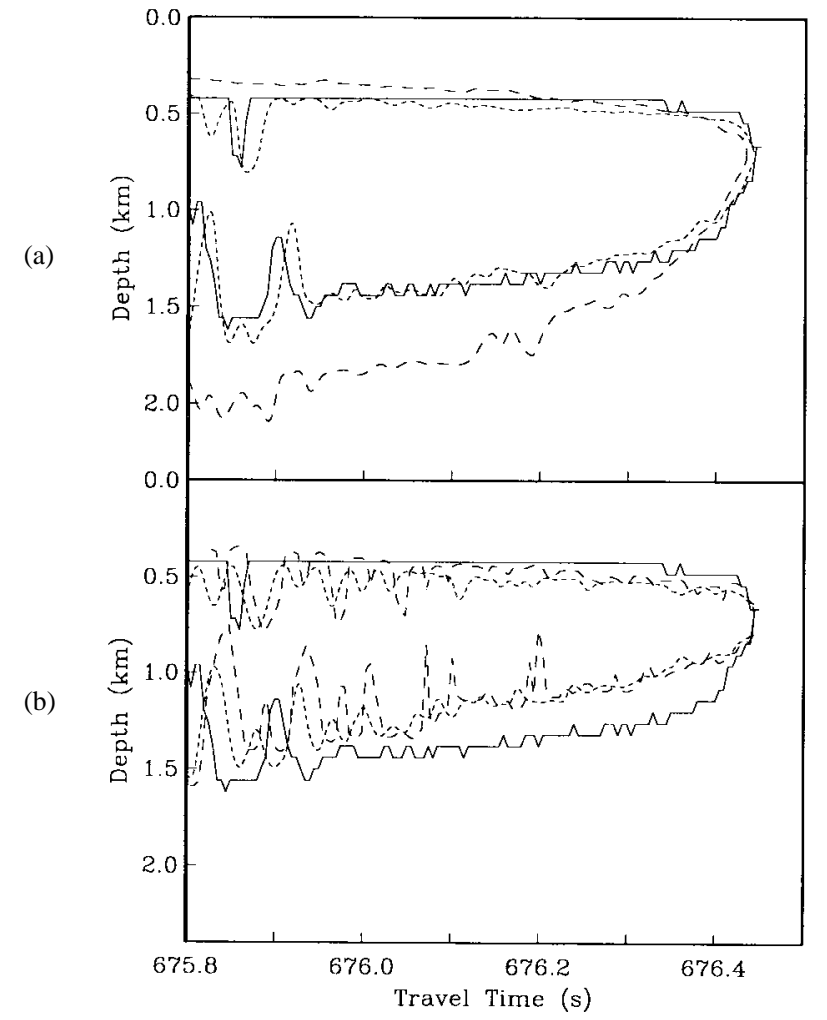

Fig. 14. Pulse envelope observed from the SLICE89 experiment (solid line) and numerical simulation calculations. (a) Monte Carlo simulation result for 2.0 GM (long-dash line) and 0.5 GM (short-dash line). (b) Numerical simulation with mesoscale perturbations (long-dash line) and numerical simulation without any perturbations (short-dash line).

Fig. 14 also shows the wavefront envelopes for the SLICE89 data and the numerical simulations at 0.5 and 2.0 GM. It is clear that the $0.5 \mathrm{GM}$ prediction matches the data very well, indicating a $0.5 \mathrm{GM}$ level near the axis.

For the ray-like arrivals, travel-time variances have been calculated for individual fronts [15]. The broad-band variance $\sigma_{b}^{2}\left(z_{u}\right)$ is a measure of travel-time variations which take place on a time scale of less than $10 \mathrm{~min}$, while the wander variance $\sigma_{w}^{2}\left(z_{u}\right)$ is a measure of the travel-time variations which take place on longer timescales $(\simeq 1 \mathrm{~h})$. Since ray travel times are very sensitive to internal wave sound-speed changes at the upper apex of the ray, [32] each wavefront segment is identified with the average upper turning depth $\left(z_{u}\right)$ of the rays that make up that front.

The internal wave displacement variance is estimated using the travel-time variances calculated from the numerical simulation at 0.5 and $2.0 \mathrm{GM}$ by assuming a linear relationship between internal wave displacement variance and travel-time variance. For the two travel-time variances, the internal wave displacement variance is calculated using

$$
\begin{aligned}
\zeta_{b, w}^{2}\left(z_{u}\right) & =A \overline{\zeta^{2}}{ }_{2.0 \mathrm{GM}}\left(z_{u}\right)+B \overline{\zeta^{2}}{ }_{0.5 \mathrm{GM}}\left(z_{u}\right) \\
A & =\frac{\left(\sigma_{b, w}^{2}\left(z_{u}\right)-\hat{\sigma}_{b, w}^{2}\left(0.5, z_{u}\right)\right)}{\left(\hat{\sigma}_{b, w}^{2}\left(2.0, z_{u}\right)-\hat{\sigma}_{b, w}^{2}\left(0.5, z_{u}\right)\right)} \\
B & =1-A
\end{aligned}
$$

where $\hat{\sigma}_{b, w}^{2}\left(0.5, z_{u}\right)$ and $\hat{\sigma}_{b, w}^{2}\left(2.0, z_{u}\right)$ are the broad-band $(b)$ and wander $(w)$ variances calculated from the numerical 


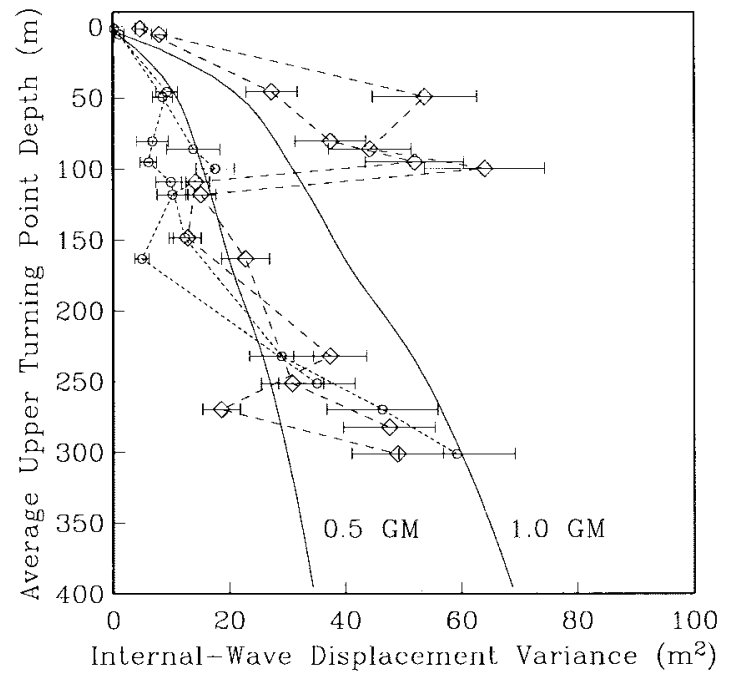

Fig. 15. SLICE89 internal wave displacement variance inferred from Monte Carlo numerical simulations. Open circles are displacements inferred from the wander variance and diamonds are displacements inferred from the broad-band variance.

simulations at 0.5 and $2.0 \mathrm{GM}$, and $\sigma_{b, w}^{2}\left(z_{u}\right)$ are the measured variances from the experiment. The quantities $\overline{\zeta^{2}}{ }_{2.0 \mathrm{GM}}\left(z_{u}\right)$ and $\bar{\zeta}^{2}{ }_{0.5 \mathrm{GM}}\left(z_{u}\right)$ are the variances of internal wave displacement at 2.0 and $0.5 \mathrm{GM}$, calculated from

$$
\begin{aligned}
\overline{\zeta^{2}}{ }_{1.0 \mathrm{GM}}(z) & =\zeta_{r}^{2} \frac{N_{G}^{2}(z)}{N_{G}^{2}\left(z_{r}\right)} \\
N_{G}^{2} & =\sum_{j=1}^{50} \sum_{k=k_{0}}^{k_{m}}\left\langle\left|G_{j}(k)\right|^{2}\right\rangle W_{j}^{2}(k, z) d k
\end{aligned}
$$

where $z_{r}$ is a reference depth at which the buoyancy frequency is $3 \mathrm{cph},\left\langle\left|G_{j}(k)\right|^{2}\right\rangle$ is the GM internal wave displacement spectrum [7] in terms of vertical mode number $j$ and horizontal wavenumber $k, \zeta_{r}=7.3 \mathrm{~m}, k_{0}=d k=1 / 32 \mathrm{cpkm}$, $k_{m}=1 \mathrm{cpkm}$, and $W_{j}(k, z)$ are solutions to the internal wave eigenmode equation [9]. Fig. 15 shows the estimates of $\zeta_{b, w}^{2}\left(z_{u}\right)$, and $\overline{\zeta^{2}}{ }_{1.0 \mathrm{GM}}\left(z_{u}\right)$ has been plotted for reference. For acoustic energy with turning points below $100 \mathrm{~m}$, the variance estimates indicate internal wave displacements which are from 0.4 to 1.0 times the reference GM level, but for energy that samples the upper $100 \mathrm{~m}$ the two different variance measures imply very different internal wave displacements. This result shows that the spectrum of sound-speed variations that existed in the ocean near the surface was significantly different from the GM spectrum modeled in the numerical simulations.

Comparing to other studies, Stoughton et al. [39] measured internal wave displacements near Bermuda. They found the standard GM level for rays which sample the upper $200 \mathrm{~m}$ of the ocean and $0.5 \mathrm{GM}$ for rays whose upper turning points sample the depth from 600 to $900 \mathrm{~m}$. The Bermuda experiment did not have a vertical array nor were the transmissions rapid enough to separate the broad-band and wander variances. Therefore, to compare our result with the Bermuda experiment, one must use the average of the wander and the broad-band variance. The average of the curves in Fig. 15 gives a similar result to the Bermuda experiment; that is, near 0.5 GM for the main thermocline and near 1.0 GM in the upper ocean. However, examining the broad-band and wander variances separately reveals the inconsistency of applying the GM model to the upper $100 \mathrm{~m}$ of the ocean. Furthermore, the use of the wavefront envelope extends the measurement of internal waves into the deep ocean, giving more vertical resolution than was achieved by Stoughton. Use of the wavefront envelope is clearly suboptimal, however, because the results depend on the precise intensity cut-off level used. Therefore, in future experiments, like the ATOC measurements, use of moderesolving arrays is essential so that modal travel times can be used as acoustic observables.

2) ATOC and AET: For internal wave tomography using the ATOC and AET data sets, the approach will be to use non-Monte Carlo methods to avoid the computational intensity associated with an extensive survey of parameter space. In contrast to the SLICE89 experiment, for the ATOC and AET experiments, it is desired to infer all of the parameters of the GM internal wave model, not just the energy $\zeta_{0}^{2}$, since it is known that the acoustic observables can be functions of the other GM parameters. For example, an increase in the internal wave energy reduces the vertical coherence, but an increase in the GM vertical modenumber bandwidth, $j_{*}$, has the same effect. Also, because of the observed weak scattering for the early ray-like arrivals of the AET (see Section II), a geometrical optics approach is adopted for the forward acoustic propagation problem.

Our focus here will be on the acoustic observables of traveltime variance, vertical coherence, and temporal coherence. The vertical and temporal coherences are key observables since they are directly related to the internal wave field vertical and temporal coherences [31]. Vertical coherence observations strongly constrain the GM parameters $j_{*}$ and $q$, and temporal coherences strongly constrain the GM parameter $p$ [see (49)]. For the parameter $j_{*}$, for example, it can be shown analytically that the approximate scaling relations follow, i.e.,

$$
\begin{aligned}
& L_{p} \propto 1 / j_{*} \\
& L_{z}^{2} \propto 1 / j_{*}^{2}
\end{aligned}
$$

and

$$
\left\{\omega^{2}\right\} \text { not a function of } j_{*}
$$

where $\left\{\omega^{2}\right\}$ is the spectrum averaged value of the square of the internal wave frequency as in the work of Flatté and Esswein [31]. The last condition simply follows from the fact that the GM spectrum is factorized in terms of mode number and frequency [8], therefore, $\left\{\omega^{2}\right\}$ is only a function of $p$. Using the expressions for temporal and depth coherence $\left(t_{0}\right.$ and $z_{0}$ ) from Flatté and Stoughton [16], it is found that

$$
\begin{aligned}
& z_{0}^{-2} \propto L_{p} / L_{z}^{2} \propto j_{*} \\
& t_{0}^{-2} \propto L_{p}\left\{\omega^{2}\right\} \propto 1 / j_{*}
\end{aligned}
$$

and

$$
\tau^{2} \propto L_{p} \propto 1 / j_{*}
$$

For the calculations of the acoustic coherence function (the forward problem), the approach of Flatté and Stoughton [16] 
(and several others) is taken and the coherence between two points $\psi(1)$ and $\psi(2)$ is written using the expression

$$
\left\langle\psi(1) \psi^{*}(2)\right\rangle=\exp (-D(1,2) / 2)
$$

where $D$, the phase-structure function, is defined as

$$
D(1,2)=\left\langle(\delta \phi(1)-\delta \phi(2))^{2}\right\rangle .
$$

Equation (43) has been obtained in many ways [1], [40]-[42], and the traditional derivations rely on the assumption that $\chi$, the log-amplitude, and $\delta \phi$, the phase fluctuation, are zero-mean Gaussian random variables with $\langle\chi(1) \chi(2)\rangle=\langle\chi \phi\rangle=0$. However, the most general derivation is due to Dashen et al. [12] using the path-integral technique. The path-integral derivation shows that (43) is very general and applies regardless of whether or not the wave fluctuations are unsaturated [12]. The limitations on this result come from: 1) the parabolic approximation to the Helmholtz equation; 2) depth and/or geographic inhomogeneity of the ocean sound-speed fluctuation field; and 3) azimuthal anisotropy of the ocean soundspeed fluctuation field. Perhaps the most restrictive of these limitations comes from violations of depth inhomogeneity.

The phase structure function $D$ becomes

$$
\begin{aligned}
D(1,2) & =\sigma^{2}\left\langle\left(\int_{\Gamma_{1}} d s_{1} \frac{\delta c}{c^{2}}-\int_{\Gamma_{2}} d s_{2} \frac{\delta c}{c^{2}}\right)^{2}\right\rangle \\
& \simeq 2 \sigma^{2}\left(\tau^{2}-\tau_{1} \tau_{2}\right)
\end{aligned}
$$

where $\Gamma_{1}$ and $\Gamma_{2}$ are ray paths terminating at points 1 and 2. The quantity $\tau^{2}$ is the mean square travel time fluctuation given by

$$
\begin{aligned}
\tau^{2} & =\left\langle\delta \phi^{2}\right\rangle / \sigma^{2} \\
& =\int_{\Gamma_{1}} d s_{1}\left[\frac{1}{c^{2}}\left(\frac{d c}{d z}\right)_{p}\right]_{1} \int_{\Gamma_{1}} d s_{2}\left[\frac{1}{c^{2}}\left(\frac{d c}{d z}\right)_{p}\right]_{2} \rho\left(s_{1}, s_{2}\right)
\end{aligned}
$$

and $\tau_{1} \tau_{2}$ is the travel-time fluctuation covariance given by

$$
\begin{aligned}
\tau_{1} \tau_{2} & =\left\langle\delta \phi_{1} \delta \phi_{2}\right\rangle / \sigma^{2} \\
& =\int_{\Gamma_{1}} d s_{1}\left[\frac{1}{c^{2}}\left(\frac{d c}{d z}\right)_{p}\right]_{1} \int_{\Gamma_{2}} d s_{2}\left[\frac{1}{c^{2}}\left(\frac{d c}{d z}\right)_{p}\right]_{2} \rho\left(s_{1}, s_{2}\right)
\end{aligned}
$$

where $\rho$ is the displacement correlation function. At this point, it has not been established that the aforementioned approach is the correct treatment of the forward problem; this is a start.

The internal wave displacement correlation function is modeled as

$$
\begin{aligned}
& \rho(\Delta x, \Delta z, \Delta t ; \bar{z}) \\
& =\left(\zeta_{0}\right)^{2}\left(N_{0} / N(\bar{z})+\sum_{n=1}^{n \max } a_{n} A_{n}(\bar{z})\right) \\
& \quad \times \operatorname{Re}\left[\sum_{j=1}^{j \max } \int_{-\infty}^{\infty} d k_{x} \int_{-\infty}^{\infty} d k_{y} \times F_{j}\left(k_{x}, k_{y}\right)\right. \\
& \left.\quad \times \exp \left(i\left(k_{x} \Delta x+k_{z}(\bar{z}, j) \Delta z-\omega\left(k_{x}, k_{y}, j\right) \Delta t\right)\right)\right]
\end{aligned}
$$

where $F_{j}\left(k_{x}, k_{y}\right)$ is the GM internal wave spectrum, in terms of vertical mode number $j$ and horizontal wavenumbers $k_{x}$ and $k_{y}$, which is given by [25]

$$
F_{j}\left(k_{x}, k_{y}\right)=M_{j} \frac{1}{\left(j^{2}+j_{*}^{2}\right)^{q}} M_{k} \frac{k_{j}^{p-2}\left(k_{x}^{2}+k_{y}^{2}\right)^{1 / 2}}{\left(k_{x}^{2}+k_{y}^{2}+k_{j}^{2}\right)^{\frac{p+1}{2}}} .
$$

Here $k_{j}=\pi j f / N_{0} B, N_{0} B=\int_{0}^{z_{b}} N(z) d z, f=2 \Omega \sin$ (latitude) is the local vertical component of the earths rotation vector, and $N(z)$ is the buoyancy frequency profile. The WKB internal wave dispersion relation $\left(\omega^{2}=f^{2}+N^{2}\left(k_{x}^{2}+\right.\right.$ $\left.\left.k_{y}^{2}\right) / k_{z}^{2}\right)$ and the WKB vertical wavenumber $\left(k_{z}(\bar{z}, j)=\right.$ $\left.\pi j N(\bar{z}) / N_{0} B\right)$ are used since they are an integral part of the GM model [1], [26]. The constants $M_{j}$ and $M_{k}$ are normalizations so that the spectrum integrates to unity. Nominal values for the GM parameters are: $q=1, p=3, j_{*}=3, N_{0}=3 \mathrm{cph}$, and $\zeta_{0}=7.3 \mathrm{~m}$, and $j \mathrm{max}$ is generally taken to be between 50 and 100 [9], [13].

The parameters to be determined from the inverse are the internal wave energy $\zeta_{0}^{2}$, the coefficients $\left\{a_{n}\right\}$ which give the corrections to WKB depth scaling for internal wave energy, the modal bandwidth $j_{*}$, and the power law exponents $p$ and $q$.

It can be readily seen that $\rho$ depends linearly on $\zeta_{0}^{2}$ and $\left\{a_{n}\right\}$ and nonlinearly on $p, q$, and $j_{*}$. Estimation of these parameters will therefore require a mixed linear/nonlinear inversion scheme. An iterative linear scheme based on Newton's method may prove effective if the nominal GM model parameters are a good first guess.

At this point, there is no analytic theory for mode travel-time spread, so there is no known analytic weighting function. Numerical simulations must be done to estimate the sensitivity of mode spread to variations in internal wave spectral parameters. In addition, there is the issue of mesoscale effects on modal spread. For SLICE89, the mesoscale field was very weak, and the pulse finale was unaffected by these perturbations. This situation may be different for the ATOC data [38].

3) Other Observables-Intensity Variability: So far, our discussion of internal wave tomography has only touched on the information in the acoustic phase, which is primarily affected by large-scale internal waves. Ewart et al. [35] has suggested doing internal wave tomography with intensity information alone since this arrangement would lead to significant experimental simplification.

In the parabolic approximation, it is convenient to use the inverse of intensity $J=1 / I$. Along a geometrical optics ray path, the following expression for $J$ has been derived [10]:

$$
\frac{d^{2} J}{d x^{2}}+\frac{J c_{0}^{2}}{c^{4}}\left[c \frac{d^{2} c}{d z^{2}}-3\left(\frac{d c}{d z}\right)^{2}\right]=0 .
$$

Letting the sound speed be a mean $c$ plus a fluctuation $\delta c$ and expanding $J=\bar{J}+J_{1}+\cdots$ in orders of $\delta c$, the results are

$$
\frac{d^{2} \bar{J}}{d x^{2}}+\frac{\bar{J} c_{0}^{2}}{c^{4}}\left[c \frac{d^{2} c}{d z^{2}}-3\left(\frac{d c}{d z}\right)^{2}\right]=0
$$

and

$$
\frac{d^{2} J_{1}}{d x^{2}}+\frac{\bar{J} c_{0}^{2}}{c^{3}} \frac{d^{2} \delta c}{d z^{2}}=0
$$


where, in (52), small terms proportional to $\delta c\left(d^{2} c / d z^{2}\right)$, $(d c / d z)(d \delta c / d z)$, and $(\delta c / c)(d c / d z)^{2}$ have been ignored. Putting (52) in integral form, squaring, and taking the expectation value yields

$$
\begin{aligned}
\left\langle J_{1}^{2}\right\rangle= & \int_{0}^{R} d x_{1} \int_{0}^{R} d x_{1}^{\prime} \int_{0}^{x_{1}} d x_{2} \int_{0}^{x_{1}^{\prime}} d x_{2}^{\prime} \bar{J}\left(x_{2}\right) \\
& \times \bar{J}\left(x_{2}^{\prime}\right)\left\langle\frac{d^{2} \delta c\left(x_{2}\right)}{d z^{2}} \frac{d^{2} \delta c\left(x_{2}^{\prime}\right)}{d z^{2}}\right\rangle
\end{aligned}
$$

Equation (53) shows that fluctuations in $J$ are related to the correlation function of sound-speed fluctuation vertical curvature, that is, observations of $J$ can give us very detailed information about the smallest scales of the internal wave field. Recall that the phase structure function $D$, which was central to the calculation of coherence, was related to the correlation function of sound-speed fluctuations rather than their gradients.

\section{SUMmARY}

Observations of basin-scale broad-band acoustic transmissions in the north Pacific with a center frequency of $75 \mathrm{~Hz}$ have surprisingly revealed that the acoustic wave fields of the early arriving wavefronts are in the unsaturated or partially saturated regime. Pulse wander is significantly larger than pulse spread, and the PDF of wave front intensity is close to the log-normal distribution. Predictions of the wave propagation regime based on $\Lambda$ and $\Phi$, where $\Lambda$ is calculated using CW assumptions, indicate saturated wave fields, in contradiction to the observations. A reasonable but simplistic modification to the $\Lambda$ calculation, which models the ray tube width for a finite bandwidth, gives values which are 1000 times smaller than the $\mathrm{CW}$ calculation and predicts the wave propagation regime to be unsaturated. A proper treatment of the ray tube width, which includes Bowlin's [14] treatment of the sound channel, but also accounts for the ray tube extension by internal waves as described by Simmen et al. [10], is a first-order WPRM issue which remains unresolved.

In general, a theory for pulse propagation is needed to understand the basin-scale observations presented here and elsewhere. The Born approximation results presented here, which do not include a treatment of the ocean waveguide, are a good starting place for this development. The Born results, however, do not describe a $10^{2}$ or $10^{3}$ magnitude effect between $\mathrm{CW}$ and broad-band cases; therefore, much remains to be discovered in explaining the AET unsaturated wavefields. Also, it must be reiterated that there are range limitations to the validity of the Born approximation, and, therefore, the arrival of a fully broad-band theory for long-range basin-scale transmissions might be very far away.

The acoustic inverse for internal wave parameters utilizing a geometrical optics approach constitutes a challenging mixed linear/nonlinear inverse. Coherence observations, along with travel time variance, constrain the inverse since vertical coherences are strongly affected by the parameters $j_{*}$ and $q$, whereas temporal coherence is strongly affected by the parameter $p$. Utilizing the information in acoustic intensity variability can provide a useful probe of small-scale internal waves, but there is very little known about the forward model for these observables for long-range ocean acoustic propagation. A Monte Carlo approach for acoustic fluctuations observed in the SLICE89 experiment showed the range-average internal wave energy to be 1.0 to 0.4 times the GM reference level for the depth range 300 to $100 \mathrm{~m}$ and about $0.5 \mathrm{GM}$ for the depth range 300 to $1000 \mathrm{~m}$. The sensitivity of this result to changes in other parameters of the GM model was not explored. For acoustic energy which sampled the upper $100 \mathrm{~m}$ of the ocean, the GM Monte Carlo runs could not be rendered consistent with the observations by simply adjusting the internal wave energy; this is a result of the well-known inadequacy of the GM model in the upper ocean. For basin-scale transmissions, it is imperative that we be able to formulate a model of upper ocean internal waves.

Observed acoustic normal mode arrivals for a center frequency of $75 \mathrm{~Hz}$ show strong mode coupling, and the coupling magnitude is consistent with numerical simulations using GM sound-speed fluctuations. The strong mode coupling signal from internal waves could, in principle, be used in an internal wave inverse, but the only way to solve the forward problem is through computationally intensive Monte Carlo runs [25], [43]. The large mode coupling at $75 \mathrm{~Hz}$ hinders efforts to increase the vertical resolution of acoustic thermometry. Computer simulations at $28 \mathrm{~Hz}$ suggest the first few modes could be treated adiabatically and, therefore, could be used in acoustic thermometry.

\section{ACKNOWLEDGMENT}

The author wishes to thank T. Duda, J. Lynch, T. Stanton, D. Chu, and S. Flatté for many useful discussions concerning internal waves and acoustic fluctuations.

\section{REFERENCES}

[1] S. Flatté, R. Dashen, W. Munk, K. Watson, and F. Zachariasen, Sound Transmission Through a Fluctuating Ocean. Cambridge, U.K.: Cambridge Univ., 1979.

[2] S. M. Flatté, "Wave propagation through random media: Contributions from ocean acoustics," Proc. IEEE, vol. 71, pp. 1267-1294, 1983.

[3] F. S. Henyey and C. Macaskill, "Sound through the internal wave field," in Stochastic Modeling in Physical Oceanography. Boston, MA: Birkhauser, 1996, pp. 141-184.

[4] W. Munk, P. Worcester, and C. Wunsch, Ocean Acoustic Tomography. Cambridge, U.K.: Cambridge Univ. Press, 1995.

[5] B. D. Dushaw, B. M. Howe, J. A. Mercer, R. C. Spindel, and the ATOC Group, "Multimegameter-range acoustic data obtained by bottommounted hydrophone arrays for measurement of ocean temperature," this issue, pp. 203-215.

[6] P. F. Worcester, B. D. Cornuelle, M. A. Dzieciuch, W. H. Munk, B. M. Howe, J. A. Mercer, R. C. Spindel, J. A. Colosi, K. Metzger, T. G. Birdsall, and A. B. Baggeroer, "A test of basin-scale acoustic thermometry using a large-aperture vertical array at 3250-km range in the eastern North Pacific Ocean," J. Acoust. Soc. Amer., to be published.

[7] C. Garrett and W. Munk, "Space-time scales of ocean internal waves," Geophys. Fluid Dynamics, vol. 2, pp. 225-264, 1972.

[8] W. Munk, "Internal waves and small scale processes," The Evolution of Physical Oceanography. Cambridge, MA: MIT Press, 1981, pp. 264-291.

[9] J. A. Colosi, S. M. Flatté, and C. Bracher, "Internal-wave effects on 1000-km oceanic acoustic pulse propagation: Simulation and comparison to experiment," J. Acoust. Soc. Amer., vol. 96, pp. 452-468, 1994.

[10] J. Simmen, S. M. Flatté, and G.-Y. Wang, "Wavefront folding, chaos, and diffraction for sound propagation through ocean internal waves," $J$. Acoust. Soc. Amer., vol. 102, pp. 239-255, 1997.

[11] T. F. Duda and J. B. Bowlin, "Ray-acoustic caustic formation and timing effects from ocean sound-speed relative curvature," J. Acoust. Soc. Amer., vol. 96, pp. 1033-1046, 1994. 
[12] R. Dashen, S. M. Flatté, and S. A. Reynolds, "Path-integral treatment of acoustic mutual coherence functions for rays in a sound channel," $J$. Acoust. Soc. Amer., vol. 77, pp. 1716-1722, 1985.

[13] J. A. Colosi, E. K. Scheer, S. M. Flatté, B. D. Cornuelle, M. A. Dzieciuch, W. H. Munk, P. F. Worcester, B. M. Howe, J. A. Mercer, R. C. Spindel, K. Metzger, T. G. Birdsall, and A. B. Baggeroer, "Comparisons of measured and predicted acoustic fluctuations for a $3250-\mathrm{km}$ range propagation experiment in the eastern North Pacific Ocean," J. Acoust. Soc. Amer., to be published.

[14] J. B. Bowlin, "Generating eigenray tubes from two solutions of the wave equation," J. Acoust. Soc. Amer., vol. 89, pp. 2663-2669, 1991.

[15] T. F. Duda, S. M. Flatté, J. A. Colosi, B. D. Cornuelle, J. A. Hildebrand, W. S. Hodgkiss, P. F. Worcester, B. M. Howe, J. A. Mercer, and R. C. Spindel, "Measured wave-front fluctuations in 1000-km pulse propagation in the Pacific Ocean," J. Acoust. Soc. Amer., vol. 92, no. 2, pp. 939-955, 1992.

[16] S. M. Flatté and R. B. Stoughton, "Predictions of internal-wave effects on ocean acoustic coherence, travel time variance, and intensity moments for very long-range propagation," J. Acoust. Soc. Amer., vol. 84 pp. 1414-1424, 1988.

[17] M. Marians, "Computed scintillation spectra for strong turbulence (in a thin layer)," Radio Science, vol. 10, pp. 115-119, 1975.

[18] R. L. Fante, "Electromagnetic beam propagation in turbulent media," Proc. IEEE, vol. 63, pp. 1669-1692, 1975.

[19] W. H. Munk and F. Zachariasen, "Sound propagation through a fluctuating stratified ocean: Theory and observation," J. Acoust. Soc. Amer. vol. 59, pp. 818-838, 1976.

[20] S. M. Flatté, G. Y. Wang, and J. Martin, "Irradiance variance of optical waves through atmospheric turbulence by numerical simulation and comparison with experiment," J. Opt. Soc. Amer., vol. 10, pp. $2363-2370,1993$

[21] C. T. Tindle and K. M. Guthrie, "Rays as interfering modes in underwater acoustics," J. Sound Vib., vol. 34, pp. 291-295, 1974.

[22] K. M. Guthrie and C. T. Tindle, "Ray effects in the normal mode approach to underwater acoustics," J. Sound Vib., vol. 47, pp. 403-413, 1976.

[23] S. M. Flatté, C. Bracher, and G.-Y. Wang, "Probability-density functions of irradience for waves in atmospheric turbulence calculated by numerical simulation," J. Opt. Soc. Amer., vol. 11, pp. 2080-2092, 1994.

[24] W. Munk and C. Wunsch, "Observing the oceans in the 1990s," Phil. Trans. R. Soc. Lond., vol. 307, pp. 439-464, 1982.

[25] J. A. Colosi and S. M. Flatté, "Mode coupling by internal waves for multi-megameter acoustic propagation in the ocean," J. Acoust. Soc. Amer., vol. 100, pp. 3607-3620, 1996.

[26] J. A. Colosi and M. G. Brown, "Efficient numerical simulation of stochastic internal wave induced sound speed perturbation fields," $J$. Acoust. Soc. Amer., vol. 103, pp. 2232-2235, 1998

[27] B. Headrick, "Analysis of internal wave induced mode coupling effects on the 1995 SWARM experiment acoustic transmissions," Ph.D. dissertation, WHOI, Woods Hole, MA, p. 145, 1997.

[28] W. H. Munk, "Once again: Once again-Tidal friction," Prog. Oceanogr., pp. 162-168, 1997.

[29] S. A. Thorpe, "The excitation, dissipation and interaction of internal waves in the deep water," J. Geophys. Res., vol. 80, pp. 328-338, 1975.

[30] W. Munk, P. Worcester, and F. Zachariasen, "Scattering of sound by internal wave currents: The relation to vertical momentum flux," J. Phys.
Oceanogr., vol. 11, pp. 442-454, 1981.

[31] S. Flatté, "Principles of acoustic tomography of internal waves," Proc. IEEE, vol. 71, pp. 372-377, 1983.

[32] S. M. Flatté and R. B. Stoughton, "Theory of acoustic measurement of internal wave strength as a function of depth, horizontal position, and time," J. Geophys. Res., vol. 91, pp. 7709-7720, 1986.

[33] B. J. Uscinski, "Acoustic scattering by ocean irregularities: Aspects of the inverse problem," J. Acoust. Soc. Amer., vol. 79, pp. 347-355, 1986.

[34] J. A. Colosi, "Random media effects in basin-scale acoustic transmissions," in Proc. 1997 Aha Hulikoá Winter Workshop, 1997, pp. 157-166.

[35] T. E. Ewart, S. A. Reynolds, and D. Rouseff, "Determining an ocean internal wave model using acoustic log-amplitude and phase-A Rytov inverse," J. Acoust. Soc. Amer., vol. 104, pp. 146-155, 1998.

[36] P. F. Worcester, B. D. Cornuelle, J. A. Hildebrand, W. S. Hodgkiss Jr., T. F. Duda, J. Boyd, B. M. Howe, J. A. Mercer, and R. C. Spindel, "A comparison of measured and predicted broadband acoustic arrival patterns in travel time-depth coordinates at 1000-km range," J. Acoust. Soc. Amer., vol. 95, pp. 3118-3128, 1994.

[37] B. D. Cornuelle, P. F. Worcester, J. A. Hildebrand, W. S. Hodgkiss Jr., T F. Duda, J. Boyd, B. M. Howe, J. A. Mercer, and R. C. Spindel, "Ocean acoustic tomography at $1000-\mathrm{km}$ range using wavefronts measured with a large aperture vertical array," J. Geophys. Res., vol. 98, pp. 365-377, 1993.

[38] J. L. Spiesberger and F. D. Tappert, "Kaneohe acoustic thermometer further validated with rays over $3700 \mathrm{~km}$ and the demise of the idea of axially trapped energy," J. Acoust. Soc. Amer., vol. 99, pp. 173-184, 1996.

[39] R. B. Stoughton, S. M. Flatté, and B. M. Howe, "Acoustic measurements of internal-wave rms displacement and rms horizontal current off Bermuda in late 1983," J. Geophys. Res., vol. 91, pp. 7721-7732, 1986.

[40] A. Ishimaru, Wave Propagation and Scattering in Random Media. New York: Academic, 1978

[41] B. Uscinski, The Elements of Wave Propagation in Random Media. New York: McGraw-Hill, 1977.

[42] V. I. Tatarskii, The Effects of a Turbulent Atmosphere on Wave Propagation. Israel Program for scientific translation, 1971.

[43] K. D. Heaney and W. A. Kuperman, "Very long-range source localization with a small vertical array," J. Acoust. Soc. Amer., vol. 104, pp. 2144-2159, 1998.

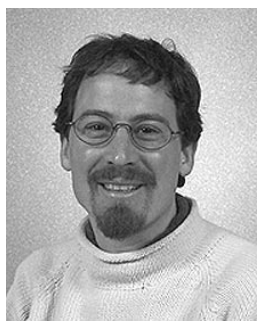

John A. Colosi received the B.S. degree in physics from the University of California at San Diego in 1988 and the Ph.D. degree in physics from the University of California at Santa Cruz in 1993.

In 1994, he returned to Scripps Institution of Oceanography, San Diego, CA, to work with Dr Peter Worcester and Prof. Walter Munk on the Acoustic Thermometry of Ocean Climate (ATOC) program. Since 1996, he has been with the faculty at the Woods Hole Oceanographic Institution Woods Hole, MA, in the Applied Ocean Physics and Engineering Department. His research interests are in ocean acoustic wave propagation through random media, coastal and deep ocean internal waves, and parallel computing. 